\title{
THE DIRECTED FOREST \\ COMPLEX OF CAYLEY GRAPHS
}

by

Kennedy Courtney

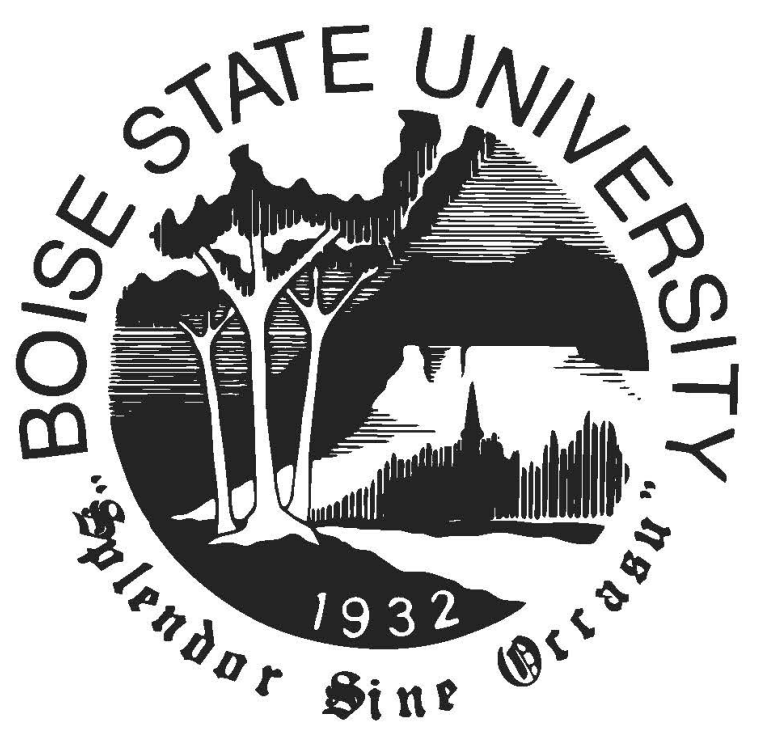

A thesis

submitted in partial fulfillment

of the requirements for the degree of

Master of Science in Mathematics

Boise State University

May 2020 
(C) 2020

Kennedy Courtney

ALL RIGHTS RESERVED 
BOISE STATE UNIVERSITY GRADUATE COLLEGE

DEFENSE COMMITTEE AND FINAL READING APPROVALS

of the thesis submitted by

Kennedy Courtney

Thesis Title: The Directed Forest Complex of Cayley Graphs

Date of Final Oral Examination: 6 March 2020

The following individuals read and discussed the thesis submitted by student Kennedy Courtney, and they evaluated her presentation and response to questions during the final oral examination. They found that the student passed the final oral examination.

Jens Harlander, Ph.D. Chair, Supervisory Committee

Uwe Kaiser, Ph.D. Member, Supervisory Committee

Zachariah Teitler, Ph.D. Member, Supervisory Committee

The final reading approval of the thesis was granted by Jens Harlander, Ph.D., Chair of the Supervisory Committee. The thesis was approved by the Graduate College. 
To all those who struggle through math, but persist. 


\section{ACKNOWLEDGMENTS}

I would like to express my deepest appreciation to my advisor, Dr. Jens Harlander, whose vision and patience gave rise to an excellent research experience. I would also

like to acknowledge my committee for their sharp eyes and sharp minds in the editing process of this thesis.

Thank you to my family for always believing and investing in me, my friends from Portland for giving long distance support, my community here at Boise State for sharing the joys and frustrations of graduate school with me, and my partner, Rahul, for his endless love and support throughout the process.

In addition, I would like to thank Dr. Christopher R. Lee, without whose guidance I would not have pursued graduate school. Thank you for meeting me where I was at without judgement, sharing opportunities to learn, and being a model of vulnerability. Your mentorship is invaluable element in my evolution, and I am wholly grateful for it. 


\begin{abstract}
Let $\Gamma$ be a directed graph. The directed forest complex, $\mathrm{DF}(\Gamma)$, is a simplicial complex whose vertices are the edges of $\Gamma$ and whose simplices are sets of edges that form a directed forest in $\Gamma$. We study the directed forest complex of Cayley graphs of finite groups. The homology of $\mathrm{DF}(\Gamma)$ contains information about the graph, $\Gamma$ and about the group, $G$. The ultimate goal is to classify $\mathrm{DF}(\Gamma)$ up to homotopy, compute its homology, and interpret the findings in terms of properties of $\Gamma$. In this thesis, we present progress made toward this goal.
\end{abstract}




\section{TABLE OF CONTENTS}

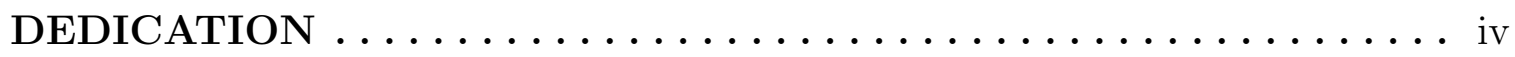

ACKNOWLEDGMENTS $\ldots \ldots \ldots \ldots \ldots \ldots \ldots \ldots \ldots \ldots$ v

Abstract $\ldots \ldots \ldots \ldots \ldots \ldots \ldots \ldots \ldots \ldots \ldots \ldots \ldots \ldots \ldots \ldots \ldots \ldots \ldots$

List of Figures $\ldots \ldots \ldots \ldots \ldots \ldots \ldots \ldots \ldots \ldots \ldots \ldots \ldots \ldots \ldots \ldots \ldots \ldots$

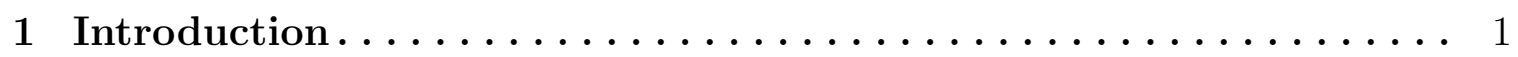

2 Simplicial Complexes $\ldots \ldots \ldots \ldots \ldots \ldots \ldots \ldots \ldots \ldots$

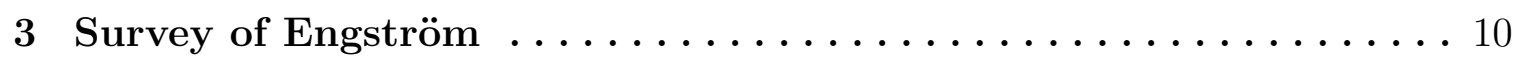

4 The Directed Forest Complex of a Cayley Graph . . . . . . . . . 22

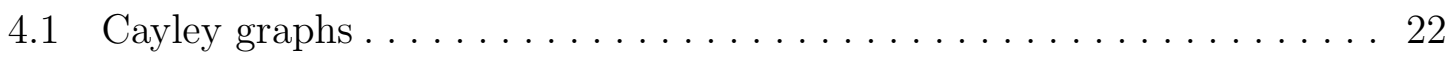

4.2 The question of purity $\ldots \ldots \ldots \ldots \ldots \ldots \ldots \ldots \ldots \ldots \ldots \ldots \ldots \ldots \ldots$

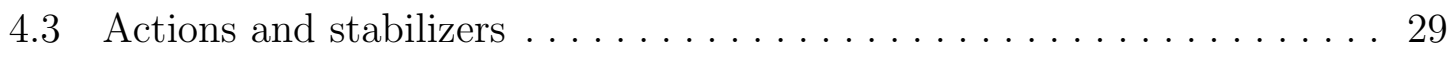

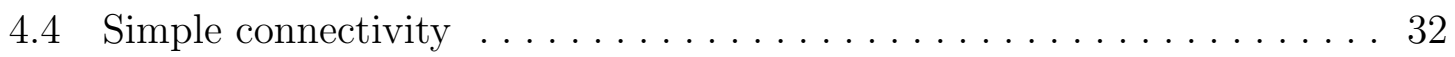

4.5 A fundamental domain ......................... 33

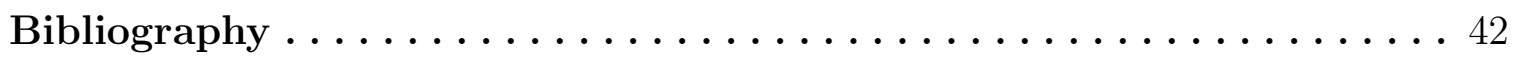




\section{LIST OF FIGURES}

2.1 A geometric realization of the simplicial complex $\Delta \ldots \ldots \ldots \ldots$

2.2 A non-example of the geometric realization of a simplicial complex. . . 6

2.3 1-dimensional, 2-dimensional, and 3-dimensional pure, shellable complexes............................ 7

2.4 A 1-dimensional connected simplicial complex with a shelling order. . . 7

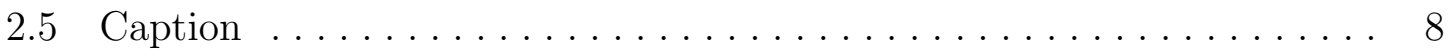

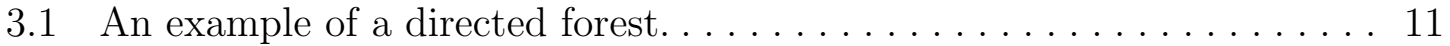

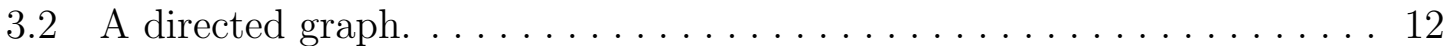

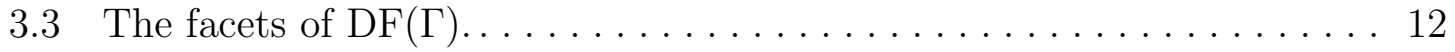

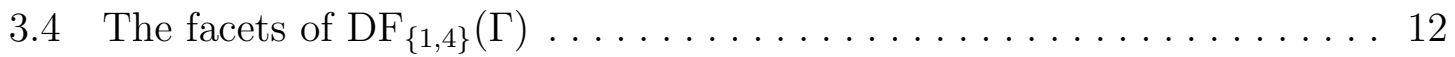

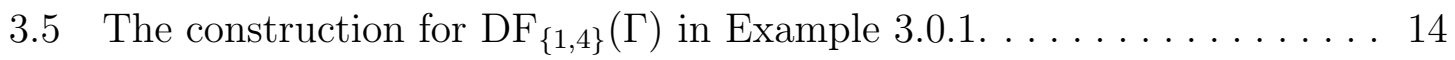

3.6 The facet $F=T_{1} \cup T_{2} \cup \cdots \cup T_{n} \in \mathrm{DF}(\Gamma)$ and $r_{i}$, the root of $T_{i}$, for every $i 19$

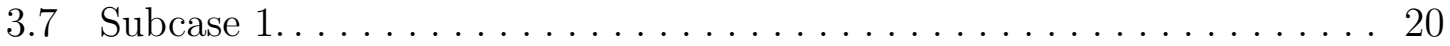

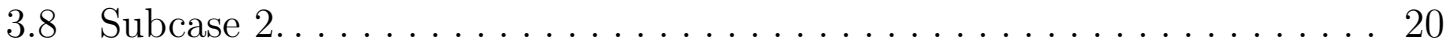

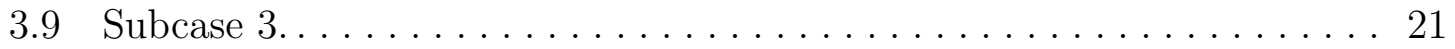

4.1 General edge in a Cayley graph $\ldots \ldots \ldots \ldots \ldots \ldots \ldots \ldots \ldots$

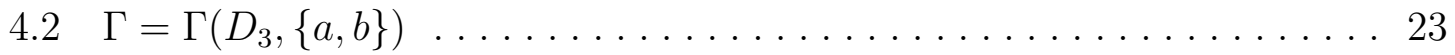

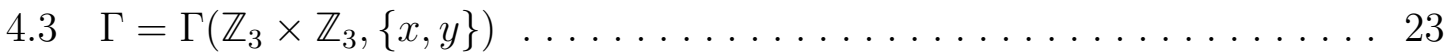

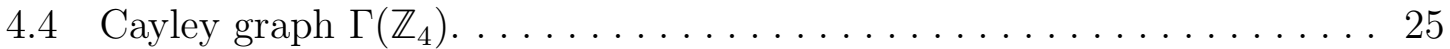




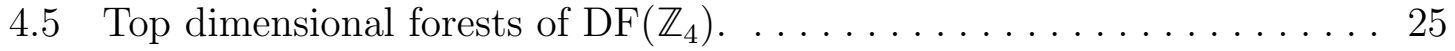

4.6 The geometric realization of $\mathrm{DF}\left(\mathbb{Z}_{4}\right) \ldots \ldots \ldots \ldots \ldots \ldots \ldots$

4.7 The Cayley graph of $D_{3}=\left\langle a, b \mid a^{2}, b^{2},(a b)^{3}\right\rangle \ldots \ldots \ldots \ldots \ldots$

$4.8 \Gamma$, the Cayley graph for $G=\mathbb{Z}_{2} \times Z_{2}=\left\langle x, y \mid x^{2}, y^{2},(x y)^{2}\right\rangle \ldots \ldots \ldots$

$4.9 \mathrm{DF}_{x y}(\Gamma) \mathrm{DF}_{y}(\Gamma) \mathrm{DF}_{1}(\Gamma), \mathrm{DF}_{x}(\Gamma) \ldots \ldots \ldots \ldots \ldots \ldots \ldots \ldots \ldots \ldots \ldots \ldots \ldots \ldots \ldots$

4.10 Some identifications made on the $\operatorname{setDF}_{x y}(\Gamma) \mathrm{DF}_{y}(\Gamma) \mathrm{DF}_{1}(\Gamma), \mathrm{DF}_{x}(\Gamma) . \quad 37$

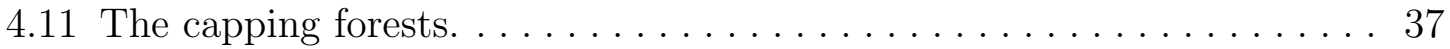




\section{Chapter 1}

\section{INTRODUCTION}

One defines a directed tree as a directed graph that contains a vertex $r$ (a root) so that every other vertex $v$ can be reached by a unique directed path from $r$ to $v$. A directed forest is a disjoint union of directed trees. Given a directed graph $\Gamma$, the directed forest complex $\operatorname{DF}(\Gamma)$ (a simplicial complex) is defined in the following way: the vertices of $\operatorname{DF}(\Gamma)$ are the edges of $\Gamma$, and a set of edges of $\Gamma$ is a simplex of $\operatorname{DF}(\Gamma)$ if the edges form a directed forest in $\Gamma$. The idea of a directed forest complex is due to Richard Stanley (see Koslov [7]). The homology of $\mathrm{DF}(\Gamma)$ contains information about the graph, for example every directed Hamiltonian cycle in $\Gamma$ gives rise to a top dimensional homology element. So the homology can "see" Hamiltonian cycles.

In this thesis, we study the directed forest complex of a Cayley graph $\Gamma$ coming from a finite group. We note that the question of whether Cayley graphs contain Hamiltonian paths or cycles is a long standing open problem. See Curran-Gallian [4] and also Pak-Radoicic [8]. The starting point of this thesis is a result of Engström [5] that says that if $\Gamma$ is an acyclic graph (and hence unlike a Cayley graph), that is a directed graph that does not contain directed cycles, then $\mathrm{DF}(\Gamma)$ is shellable, see

definition 2.0.2. In particular $\operatorname{DF}(\Gamma)$ has the homotopy type of a wedge of spheres (in various dimensions). Part of this thesis is a careful reworking of Engströms result.

Here is a summary of our main results. We show that if $\Gamma$ is a Cayley graph of 
a group $G$ then $\operatorname{DF}(\Gamma)$ might or might not be pure (i.e. all maximal simplices have the same dimension). In either case we find that its dimension is $|G|-2$. If $|G| \geq 16$ then $\operatorname{DF}(\Gamma)$ is simply connected. Using a computer, we compute the homology of some examples. One way to understand the homotopy type of $\mathrm{DF}(\Gamma)$ is by explicitely constructing a shelling order. A daunting task. Another route is to construct a good cover. In case $\operatorname{DF}(\Gamma)$ is pure we produce a fundamental domain $\operatorname{DF}_{1}(\Gamma)$ for the action of $G$ on $\operatorname{DF}(\Gamma)$ (induced by the action of $G$ on the Cayley graph $\Gamma$ ) and show (using Engström [5]) that $\mathrm{DF}_{1}(\Gamma)$ is shellable and contractible. This yields a nice decomposition

$$
\operatorname{DF}(\Gamma)=\bigcup_{g \in G} \operatorname{DF}_{g}(\Gamma)
$$

where each translate $g \mathrm{DF}_{1}(\Gamma)=\mathrm{DF}_{g}(\Gamma)$ is shellable and contractible. We tried to use this union to compute the homology of $\mathrm{DF}(\Gamma)$ using the Mayer-Vietoris spectral sequence. This entails understanding the nerve of union on one hand, and intersections of the union members on the other hand (in order to understand the coefficient system). We had success with the first part, but could not finish the second. We proved that if $\Gamma$ contains a Hamiltonian cycle, then the nerve is $\partial \Delta(G)$, the boundary of the full simplex on vertex set $G$. Topologically this is a sphere of dimension $|G|-2$.

Future goals are:

1. Understand purity of $\mathrm{DF}(\Gamma)$.

2. Understand shellability of $\mathrm{DF}(\Gamma)$.

3. Complete the computation of the homology of $\mathrm{DF}(\Gamma)$ for Cayley graphs in case $\mathrm{DF}(\Gamma)$ is pure; the major part here is to understand how the $\mathrm{DF}_{g}(\Gamma)$ intersect. 
4. Interpret the homology in terms of properties of $\Gamma$ and $G$. Is there a way to decide which top-dimensional homology elements of $H_{*}(\mathrm{DF}(\Gamma))$ come from Hamiltonian cycles?

We have tried to make this thesis as self contained as possible. A good standard reference for all matters concerning combinatorial topology is Koslov [6]. Background on graph theory can be found in Bollobas [2]. 


\section{Chapter 2}

\section{SIMPLICIAL COMPLEXES}

Because the directed forest complex is a simplicial complex, we first define the notion of a simplicial complex.

Definition 2.0.1. An (abstract) simplicial complex, $\Delta$, on set of vertices, $V$, is a set of finite subsets of $V$, generally called faces, which has the following property:

- If $\sigma \in \Delta$ and $\tau \subseteq \sigma$, then $\tau \in \Delta$.

Maximal faces in a simplicial complex are called facets. If $\sigma=\left\{v_{0}, \ldots, v_{k}\right\}$ is a face then the dimension of $\sigma$ is $k$. The dimension of a simplicial complex $\Delta$ is the maximal dimension that occurs as the dimension of a facet.

Example 2.0.1. It is generally true that the set of facets determines the simplicial complex. Let $V=\{a, b, c, d, e, f, g, h, i, j, k, l, m, n, o, p, q, r\}$ and let $\Delta$ be the simplicial complex whose set of facets is

$$
\begin{gathered}
\{\{a, b, c, d\},\{d, e, f\},\{e, f, g\},\{f, h, k\},\{h, j\},\{i, j\} \\
\{i, h\},\{k, l\},\{l, m\},\{o, p, r\},\{p, q\},\{n\}\}
\end{gathered}
$$

The geometric realization an abstract simplicial complex is defined in the following way: Place the vertices into some high dimensional $\mathbb{R}^{N}$ so that if $\sigma$ is a face of 


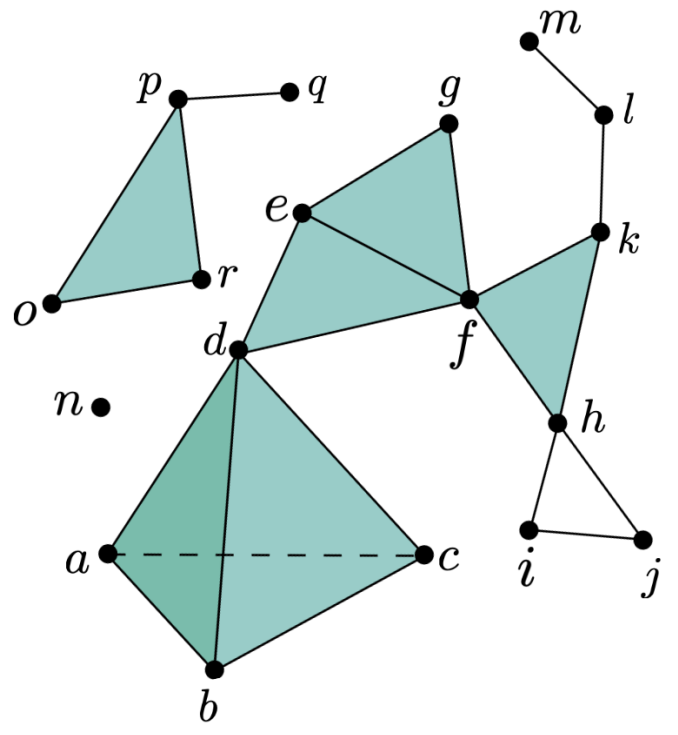

Figure 2.1: A geometric realization of the simplicial complex $\Delta$

dimension $k$, then the convex hull of the corresponding points in $\mathbb{R}^{N}$ is a geometric simplex of dimension $k$. We denote that simplex by $|\sigma|$. Let

$$
|\Delta|=\bigcup_{\sigma \in \Delta}|\sigma|
$$

$|\Delta|$ is called the geometric realization of the abstract simplicial complex $\Delta$.

Example 2.0.2. The figure 2.1 shows a geometric realization of the simplicial complex $\Delta$ given in the previous example.

$|\Delta|$ is a union of geometric simplices, so that any two simplices either do not intersect or intersect in a common subsimplex. Not every union of simplices is a geometric simplicial complex. See figure 2.2.

Definition 2.0.2. An $n$-dimensional simplicial complex is pure if all its facets are $n$-dimensional. A pure simplicial complex is shellable if its facets can be ordered 


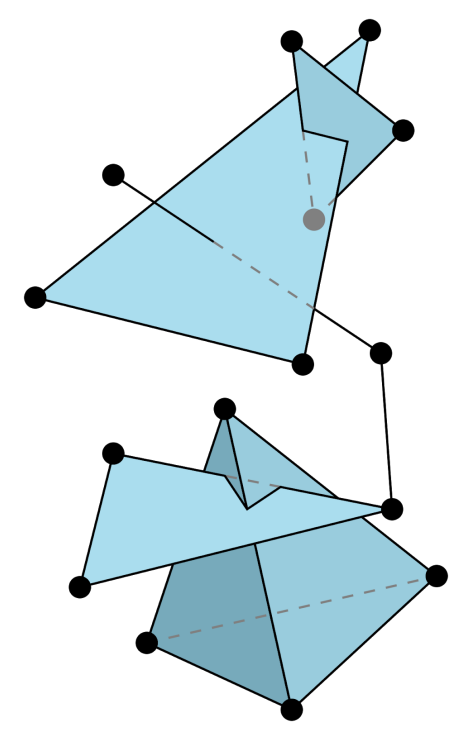

Figure 2.2: A non-example of the geometric realization of a simplicial complex.

$\left(F_{1}, \ldots, F_{k}\right)$ (referred to as shelling order) so that $\left(F_{1} \cup \cdots \cup F_{i-1}\right) \cap F_{i}$ is a non-empty union of $(n-1)$-dimensional faces, for every $i$. See figure 2.3 .

Example 2.0.3. Any 1-dimensional connected simplicial complex is shellable. Consider figure 2.4, a 1-complex with indicated shelling order.

At each step in the ordering, $\left(F_{1} \cup \cdots \cup F_{i-1}\right) \cap F_{i}$ is dimension 0 (a vertex or vertices). Notice, $F_{5}$ intersects the union of the previous facets in its entire boundary. We call this a capping facet because it introduces a cycle. $F_{6}$ is also a capping facet. The facets that are not capping facets form a maximal tree. We can collapse it to a point and obtain the wedge two 1-spheres. This example generalizes to show the following theorem.

Theorem 2.0.1. Any connected, simplicial 1-complex is shellable and is homotopy equivalent to a wedge of 1-spheres. The number of 1-spheres is exactly the number of capping facets. 


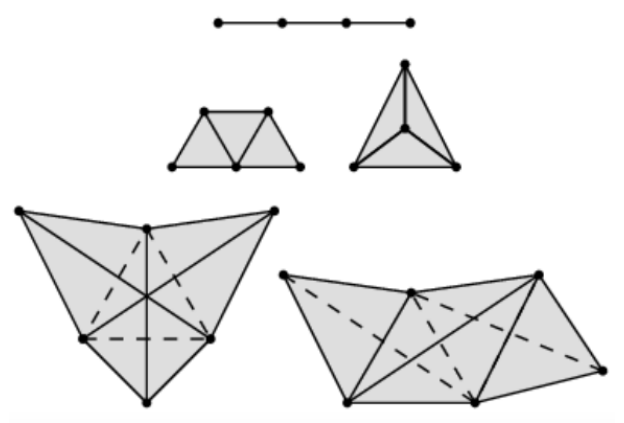

Figure 2.3: 1-dimensional, 2-dimensional, and 3-dimensional pure, shellable complexes.

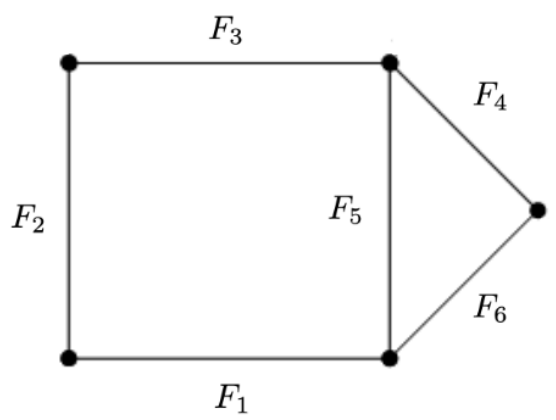

Figure 2.4: A 1-dimensional connected simplicial complex with a shelling order. 


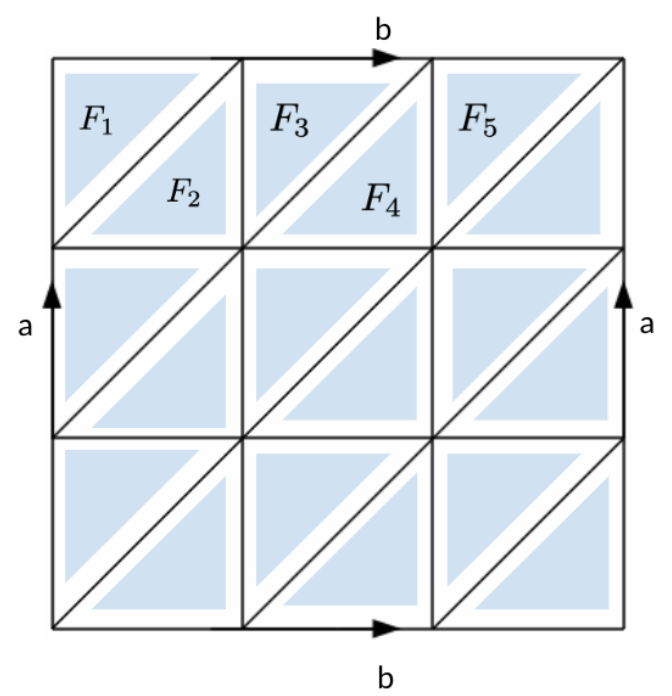

Figure 2.5: Caption

Example 2.0.4. The torus is not shellable. Consider the following simplicial complex, with edge identifications made. We will attempt to put a shelling order on this complex as follows in figure 2.5.

Notice $F_{5}$ intersects the union of the previous facets in an edge and a vertex. A vertex is not a 1-dimensional space, thus, this is not a shelling order for a torus. In fact, no shelling order exists, and the following theorem holds.

Theorem 2.0.2. If an n-dimensional pure simplicial complex is shellable, then it is homotopy equivalent to a wedge of $n$-spheres. The number of $n$-spheres is exactly the number of capping facets (there could be none, in which case the complex is homotopic to a point).

A detailed proof can be found in Koslov [6]. Nevertheless, here is a sketch of the proof.

Proof. Let $\left(F_{1}, F_{2}, \ldots, F_{k}\right)$ be a shelling order for $\Delta$. The proof uses two observations: 
1. The union of $(n-1)$-dimensional faces of an $n$-simplex which is not all of the boundary is contractible;

2. The union $\Delta_{1} \cup \Delta_{2}$ of two contractible complexes is contractible in case the intersection $\Delta_{1} \cap \Delta_{2}$ is contractible.

Let $F_{i_{1}}, \ldots, F_{i_{l}}$ be exactly the facets so that

$$
\left(F_{i_{1}} \cup \cdots \cup F_{i_{j-1}}\right) \cap F_{i_{j}}
$$

is the entire boundary of $F_{i_{j}}$. Let $\Delta_{0}$ be the union of these special facets. Using the two observations above one can show that $\Delta-\Delta_{0}$ is contractible. Contracting the subcomplex $\Delta_{0}$ in $\Delta$ results in a space that is homotopy equivalent to $\Delta$ and is a wedge of $l n$-spheres.

Q.E.D. 


\section{Chapter 3}

\section{SURVEY OF ENGSTRÖM}

This chapter presents some of the main results of Engström [5]. It does not contain original material but in places provides more detailed arguments.

Definition 3.0.1. A directed graph $\Gamma=(V, E, i, t)$ consists of sets $V, E$, and maps $i: E \rightarrow V$, and $t: E \rightarrow V$. We refer to $V$ as vertices, $E$ as edges, $i(e)$ as the initial vertex of $e$ and $t(e)$ as the terminal vertex of $e$. We think of the edge $e$ as directed from $i(e)$ to $t(e)$.

If $e$ is an edge and $i(e)=x, t(e)=y$ we often write sometimes write $e=(x \rightarrow y)$. Graphs can be drawn in the plane: single out a point in the plane for each vertex and draw a directed arc for every edge, connecting $i(e)$ to $t(e)$, putting an arrow on the arc to indicate the direction. Some arcs might cross at points that are not vertices. If such crossings can be avoided we call the graph planar.

Definition 3.0.2. Given a directed graph $\Gamma$.

1. A directed tree $T$ in $\Gamma$ is a directed subgraph that has a vertex $r$ that can connected to every other vertex $v$ of $T$ by a unique directed path from $r$ to $v$. The vertex $r$ is called the root of $T$.

2. A directed forest $F$ in $\Gamma$ is the disjoint union of directed subtrees of $\Gamma$. 

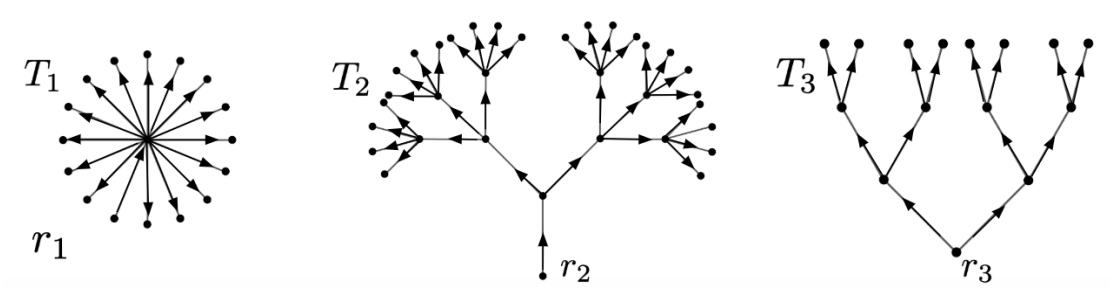

Figure 3.1: An example of a directed forest.

Definition 3.0.3. Given a directed graph $\Gamma=(V, E, i, t)$.

1. The directed forest complex of $\Gamma, \operatorname{DF}(\Gamma)$, is a simplicial complex defined in the following way: The vertex set $V$ corresponds to the set of edges in the graph, and a finite subset $\left\{e_{0}, \ldots, e_{k}\right\} \subseteq E$ is a simplex if $F=\bigcup_{i=0, \ldots, k} e_{i}$ is a directed forest in $\Gamma$.

2. Let $R \subseteq V$. Then the rooted directed forest complex, $\mathrm{DF}_{R}(\Gamma)$, is a subcomplex of $\operatorname{DF}(\Gamma)$ defined in the following way: The vertex set is $V$ and $\left\{e_{0}, \ldots, e_{l}\right\}$ is a facet if $F=\bigcup_{i=0, \ldots, l} e_{i}$ is a maximal directed forest in $\Gamma$ with root set equal to $R$.

Note that $\operatorname{DF}(\Gamma)$ is indeed a simplicial complex because every subgraph of a directed forest is a directed forest. We specified only the facets of $\mathrm{DF}_{R}(\Gamma)$. They generate the simplicial complex $\operatorname{DF}_{R}(\Gamma)$.

Example 3.0.1. Let $\Gamma$ be the directed graph in figure 3.3 .

The facets of $\mathrm{DF}(\Gamma)$ are shown in figure 3.3.

The facets of $\mathrm{DF}_{\{1,4\}}(\Gamma)$ are shown in figure 3.4.

Definition 3.0.4. If $K$ is a subcollection of a simplicial complex $\Delta$ that contains all faces of its elements, then $\mathrm{K}$ is another simplicial complex called a simplicial subcomplex. An edge $(x \rightarrow y)$ of a directed graph $\Gamma$ is nice in a subcomplex $\Delta$ of $\mathrm{DF}(\Gamma)$ if the following properties hold 


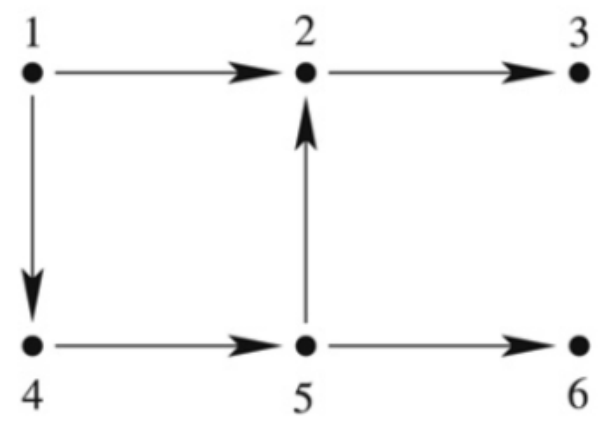

Figure 3.2: A directed graph.
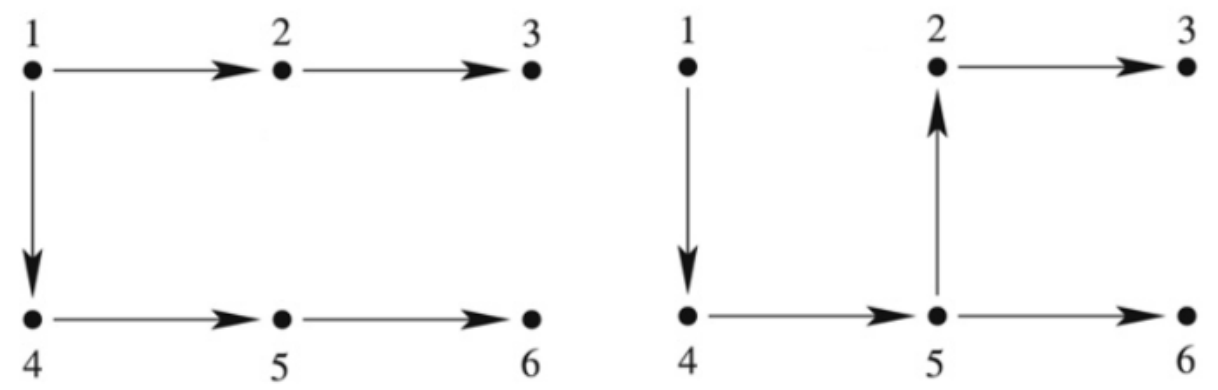

Figure 3.3: The facets of $\operatorname{DF}(\Gamma)$.
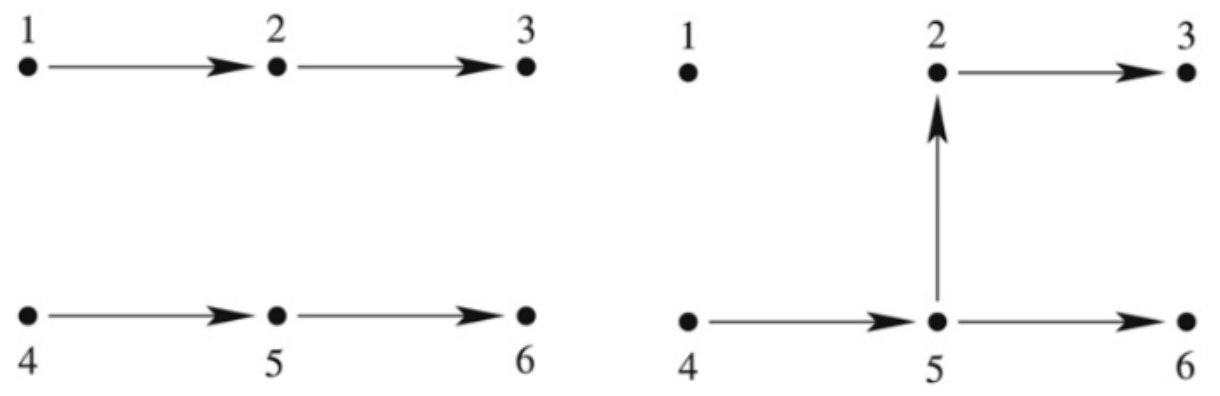

Figure 3.4: The facets of $\mathrm{DF}_{\{1,4\}}(\Gamma)$ 
1. if $\operatorname{indeg}(y)>1$, and

2. if $F \in \Delta$ is a forest without an edge directed to $y$, then $F \cup(x \rightarrow y) \in \Delta$.

Algorithm for finding nice edges. There is a general procedure for finding nice edges. We explain it using an example. Let $\Gamma$ be the directed graph in example 3.0.1. Let's find some nice edges in $\operatorname{DF}_{\{1,4\}}(\Gamma)$. We partition the vertices of the graph into left and right. A vertex is on the left if

1. the tree which contains $v$ has the same root, $r$, in all facets of $\mathrm{DF}_{\{1,4\}}$, and

2. the path from $r$ to $v$ is the same in all facets of $\mathrm{DF}_{\{1,4\}}$.

All other vertices are on the right. Then we consider the union of all the edges of the facets. Notice that this is a subgraph of $\Gamma$. As we will see, the edges which crossover the dotted line are nice.

Example 3.0.2. The construction for $\operatorname{DF}_{\{1,4\}}(\Gamma)$ in Example 3.0.1 is given in figure 3.5 .

Lemma 3.0.1. All edges crossing the dotted line in the nice construction are nice if the maximal faces have the same roots.

Proof. First, we will show that the edges can only cross the dotted line from left to right. For the sake of contradiction, assume that the edge $(z \rightarrow w)$ crosses from right to left. Since $w$ is on the left, the tree which contains $w$ has the same root $r$ in all facets and the path from $r$ to $w$ is the same in all facets. Because $w$ is on the left, the vertex directed to it is always the same since all paths from $r$ to $w$ are the same. Thus, the vertex directed to $w$ is $z$ since we know the edge $(z \rightarrow w)$ is in a maximal facet. Because all paths from $r$ to $w$ are the same, all paths from $r$ to $z$ are the same. 


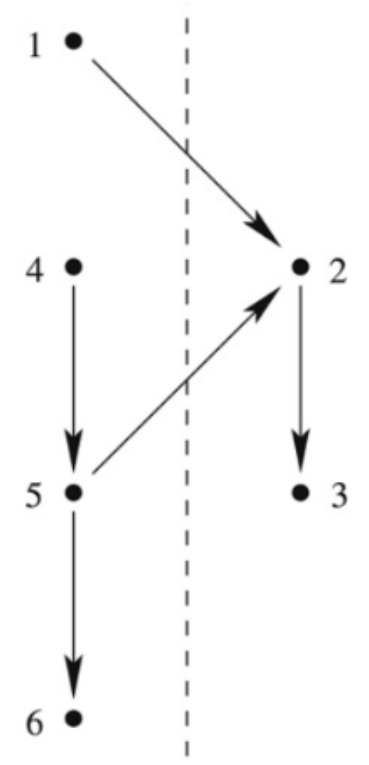

Figure 3.5: The construction for $\mathrm{DF}_{\{1,4\}}(\Gamma)$ in Example 3.0.1.

If there were more than one distinct path to $z$, then there would be more than one distinct path to $w$, which is a contradiction on our assumption. ※

Now, we will show that the first condition of niceness holds. Let the facets of the subcomplex be called $\left\{F_{i}\right\}_{i \in I}$. Assume $(x \rightarrow y)$ is an edge crossing the dotted line. Because there is an edge directed to $y, y$ is not a root. This means there must be an edge directed to $y$ in each facet. If a facet did not have an edge directed to $y$, it would not be maximal, since we know there is at least one edge directed to $y$, namely $(x \rightarrow y)$ in some facet. If $x$ were the only vertex directed to $y \in \cup_{i \in I} F_{i}$ it would be in all facets. However, in that case the path to $y$ would always be the same in all maximal facets because we know the path from $r$ to $x$ is the same in all facets, so $y$ would be on the left. Since $y$ is on the right, there must be an edge $(z \rightarrow y)$ such that $z \neq x$. This confirms that the construction satisfies condition one of niceness. Finally, we will show that condition two of niceness is satisfied. If $F \in \Delta$, and $F \cup(x \rightarrow y)$ is 
acylic, then the condition is fulfilled. A directed cycle which contains $(x \rightarrow y)$ as an edge would cross the dotted line at least twice, but all the edges crossing the dotted line go from left to right. Hence, $(x \rightarrow y)$ cannot induce a cycle, and thus, condition two is fulfilled. We can now conclude that all edges crossing the dotted line in the nice construction are nice if the maximal faces have the same roots.

Q.E.D.

Lemma 3.0.2. Let $\Gamma=(V, E, i, t)$. If $R \subseteq V$ is nonempty, and $\mathrm{DF}_{R}(\Gamma)$ has more than one facet, then there is an edge $(x \rightarrow y) \in E(\Gamma)$ which is nice in $\mathrm{DF}_{R}(\Gamma)$.

Proof. Suppose all vertices are on the left. Let $F_{1}$ and $F_{2}$ be facets of $\operatorname{DF}_{R}(\Gamma)$. We want to show that $F_{1}=F_{2}$. Let $e$ be an edge in $F_{1}$. We will show that $e$ is also in $F_{2}$. Let $v$ be terminal vertex of $e$. Then $v$ is contained in a tree $T$ of $F_{1}$ with root $r$. Let $p$ be the path $T$ that connects $r$ to $v$. Note that $p$ contains $e$. The vertex $v$ is also contained in a tree $T^{\prime}$ of $F_{2}$. Since $v$ is on the left, the root of $T^{\prime}$ is also $r$ and the path connecting $r$ to $v$ in $T_{2}$ is $p$. In particular $p$ is contained in $T_{2}$, and so, since $e$ is contained in $p, e$ is in $T_{2}$. This shows that $F_{1}$ is contained in $F_{2}$. Showing that $F_{2}$ is in $F_{2}$ can be done by the same arguments.

Q.E.D.

Here is a slightly different way to look at shellability, which also works in the non-pure setting.

Definition 3.0.5. A simplicial complex $\Delta$ is non-pure shellable if its maximal faces can be ordered $F_{1}, F_{2}, \ldots F_{n}$ such that for all $1 \leq i<k \leq n$ there are $1 \leq j<k$ and $e \in F_{k}$ such that $F_{i} \cap F_{k} \subseteq F_{j} \cap F_{k}=F_{k} \backslash\{e\}$.

Note that if $\Delta$ is pure, then this definition agrees with the first definition [1]. We give a proof. 
Theorem 3.0.3. Pure shellable as defined in Chapter 2 and non-pure shellable as defined here are equivalent if the simplicial complex in question is pure.

Proof. Assume $\Delta$ is a pure $n$-complex, and it is non-pure shellable as in Definition 3.0.5. We want to show the complex is pure shellable as in Definition 2.0.1. Let $F_{1}, \ldots, F_{m}$ be a non-pure shelling order of $\Delta$. We want to show that $\left(F_{1} \cup \cdots \cup\right.$ $\left.F_{k-1}\right) \cap F_{k}$ is the union of $(n-1)$-dimensional faces of $F_{k}$.

By the distributivity of set operations,

$$
\left(F_{1} \cup \cdots \cup F_{k-1}\right) \cap F_{k}=\left(F_{1} \cap F_{k}\right) \cup \cdots \cup\left(F_{k-1} \cap F_{k}\right) .
$$

Then there exists $1 \leq j<k$ such that $F_{1} \cap F_{k} \subseteq F_{j} \cap F_{k}=F_{k}-\{e\}$. Therefore either $F_{1} \cap F_{k}$ is $(n-1)$-dimensional or it is contained in an $(n-1)$-dimensional set in this union, in which case we remove $F_{1} \cap F_{k}$ from the union $\left(F_{1} \cap F_{k}\right) \cup \cdots \cup\left(F_{k-1} \cap F_{k}\right)$. We proceed in this fashion for $F_{2} \cap F_{k}$ up to $F_{k-1} \cap F_{k}$ to see that $\left(F_{1} \cap \cdots \cap F_{k-1}\right) \cap F_{k}$ is indeed a union of $(n-1)$-dimension faces. This show that the non-pure shellable definition implies the pure shellable definition.

Now, we will show the other direction. Assume $\Delta$ is pure, and we have a pure shelling order $F_{1}, F_{2}, \ldots, F_{m}$. Then,

$$
\begin{aligned}
\left(F_{1} \cup F_{2} \cup \cdots \cup F_{k-1}\right) \cap F_{k} & =\left(F_{1} \cap F_{k}\right) \cup \cdots \cup\left(F_{k-1} F_{k}\right) \\
& =\left(F_{i_{1}} \cap F_{k}\right) \cup \cdots \cup\left(F_{i_{h}} \cap F_{k}\right)
\end{aligned}
$$

where $\left\{i_{1}, \ldots i_{h}\right\} \subset\{1, \ldots k-1\}$ and $F_{i_{\ell}} \cap F_{k}$ is $(n-1)$-dimensional. Then, $\left(F_{i} \cap F_{k}\right) \subset$ $\left(F_{i_{\ell}} \cap F_{k}\right)=F_{k}-\{e\}$ is $(n-1)$-dimensional. Take $j=i_{\ell}$, and this shows that the pure shellability definition implies the non-pure shellability definition. 
Q.E.D.

Lemma 3.0.4. Let $F_{i}$ and $F_{k}$ be distinct facets in $\mathrm{DF}_{R}(\Gamma)$ and $(x \rightarrow y) \in F_{i} \backslash F_{k}$ a nice edge in $\mathrm{DF}_{R}(\Gamma)$. Then there is a facet $F_{j} \in \mathrm{DF}_{R}(\Gamma)$ and an edge e $\in F_{k}$ such that $F_{i} \cap F_{k} \subseteq F_{j} \cap F_{k}=F_{k} \backslash\{e\}$.

Proof. Assume $F_{i}$ and $F_{k}$ are distinct facets in $\mathrm{DF}_{R}(\Gamma)$ and $(x \rightarrow y) \in F_{i} \backslash F_{k}$ a nice edge in $\operatorname{DF}_{\mathrm{R}}(\Gamma)$. There is an edge $(z \rightarrow y) \in F_{k}$ since it is a facet and $y$ in not a root. Replace $(z \rightarrow y)$ with $(x \rightarrow y)$ to construct $F_{j} \in \mathrm{DF}_{\mathrm{R}}(\Gamma)$. Now, $F_{j} \cap F_{k}=F_{k} \backslash\{(z \rightarrow y)\}$. Since the nice edge $(x \rightarrow y) \in F_{i}$ and $(z \rightarrow y) \notin F_{i}$, we can conclude that

$$
\begin{array}{rlrl}
F_{i} \cap F_{k} & =F_{i} \cap\left(F_{k} \backslash\{(z \rightarrow y)\}\right) & & \text { (since we know } \left.(z \rightarrow y) \notin F_{i}\right) \\
& \left.\subseteq F_{k} \backslash(z \rightarrow y)\right) & \text { (by definition of intersection) } \\
& =F_{j} \cap F_{k} & \text { (by definition of } F_{j} \text { ) }
\end{array}
$$

Thus, there is a facet $F_{j} \in \operatorname{DF}_{\mathrm{R}}(\Gamma)$ and an edge $e \in F_{k}$ such that

$$
F_{i} \cap F_{k} \subseteq F_{j} \cap F_{k}=F_{k} \backslash\{e\},
$$

namely $(z \rightarrow y)$.

Q.E.D.

Theorem 3.0.5. (Engström [5]) The complex $\mathrm{DF}_{R}(\Gamma)$ is (non-pure) shellable for any $\emptyset \neq R \subseteq V(\Gamma)$.

Proof. We start by induction over the facets. Case $1 . \mathrm{DF}_{\mathrm{R}}(\Gamma)$ has only one facet. It is then a simplex and shellable. 
Case 2. $\operatorname{DF}_{R}(\Gamma)$ has more than one facet. We know that $\operatorname{DF}_{R}(\Gamma)$ has a nice edge $(x \rightarrow y)$ as show in lemma 3.0.2. Let's define $\Gamma^{\prime}, \Gamma^{\prime \prime} \subset \Gamma$ as follows,

$$
\begin{gathered}
E\left(\Gamma^{\prime}\right)=E(\Gamma) \backslash\{(z \rightarrow y) \mid z \neq x\}, \text { and } \\
E\left(\Gamma^{\prime \prime}\right)=E(\Gamma) \backslash\{x \rightarrow y\} .
\end{gathered}
$$

A facet of $\operatorname{DF}_{\mathrm{R}}(\Gamma)$ has exactly one edge to $y$ by defition of a directed tree, and thus a facet $\mathrm{DF}_{\mathrm{R}}(\Gamma)$ is contained in either $\mathrm{DF}_{\mathrm{R}}\left(\Gamma^{\prime}\right)$ or $\mathrm{DF}_{\mathrm{R}}\left(\Gamma^{\prime \prime}\right)$. Because $(x \rightarrow y)$ is nice, it is in some, but not all facets of $\operatorname{DF}_{R}(\Gamma)$. Both $\operatorname{DF}_{R}\left(\Gamma^{\prime}\right)$ and $\operatorname{DF}_{R}\left(\Gamma^{\prime \prime}\right)$ have a smaller number of facets than $\mathrm{DF}_{\mathrm{R}}(\Gamma)$, so by induction, they are shellable. Taking stock, we have a shelling order of $\mathrm{DF}_{\mathrm{R}}\left(\Gamma^{\prime}\right)$, namely, $F_{1}, F_{2}, \ldots, F_{t}$ all of which contain $(x \rightarrow y)$. We also have a shelling order of $\mathrm{DF}_{\mathrm{R}}\left(\Gamma^{\prime \prime}\right)$, that is, $F_{t+1}, F_{t+2}, \ldots, F_{t+s}$ which do not contain the nice edge. We want to show that for all $1 \leq i<k \leq s+t$ there are $1 \leq j<k$ and $(z \rightarrow w) \in F_{k}$ such that

$$
F_{i} \cap F_{k} \subseteq F_{j} \cap F_{k}=F_{k} \backslash\{z \rightarrow w\}
$$

This would show that $\operatorname{DF}_{\mathrm{R}}(\Gamma)$ is shellable where the shelling order is

$$
F_{1}, \ldots, F_{t}, F_{t+1}, \ldots, F_{t+s}
$$

Subcase 1. The inequality $1 \leq i<k \leq t$ or $t+1 \leq i<k \leq t+s$ holds. If this is true, we're done as we already showed this was shellable.

Subcase 2. The inequality $1 \leq i \leq t<k \leq s+t$ holds. We know the nice edge is in $F_{i}$, but not in $F_{k}$. Then we can construct $F_{j}$ as described in Lemma 3.0.4. The edge 

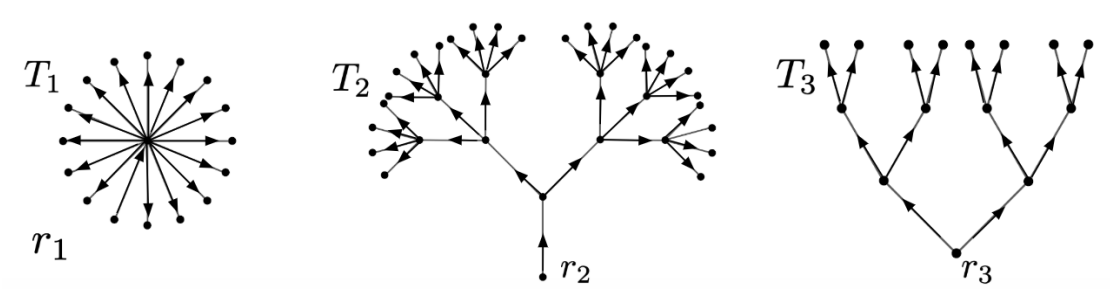

Figure 3.6: The facet $F=T_{1} \cup T_{2} \cup \cdots \cup T_{n} \in \mathrm{DF}(\Gamma)$ and $r_{i}$, the root of $T_{i}$, for every $i$

$(x \rightarrow y)$ is in $F_{j}$, so $j \leq t<k$, and we have the shelling

$$
F_{1}, \ldots, F_{t}, F_{t+1}, \ldots, F_{t+s}
$$

Q.E.D.

Corollary 3.0.6. (Engström [5]) If $\Gamma$ is a directed acyclic graph, then $\mathrm{DF}(\Gamma)$ is shellable.

Proof. Assume that $\Gamma$ is an acyclic graph, then $\Gamma$ has roots. We want to show that $\mathrm{DF}(\Gamma)=\mathrm{DF}_{\mathrm{R}}(\Gamma)$

Part 1. The inequality $\operatorname{DF}(\Gamma) \supseteq \operatorname{DF}_{\mathrm{R}}(\Gamma)$ holds: Because every facet, $F \in \operatorname{DF}_{\mathrm{R}}(\Gamma)$, with root set $R$ is a forest, $F \in \operatorname{DF}(\Gamma)$.

Part 2. The inequality $\mathrm{DF}(\Gamma) \subseteq \mathrm{DF}_{\mathrm{R}}(\Gamma)$ holds: Let $F=T_{1} \cup T_{2} \cup \cdots \cup T_{n} \in \operatorname{DF}(\Gamma)$ be a facet and let $r_{i}$ be the root of $T_{i}$, for every $i$. We can visualize this in figure 3.6

We want to show that all $r_{i} \in R$. For the sake of contradiction, assume that $r_{1} \notin$ $\mathrm{R}$. If it is not in the rootset, there is an edge which terminates at $r_{1}$. Let's call this vertex $v_{1}$.

Subcase 1: There are no other vertices terminating at $v_{1}$. As shown in figure 3.7. 

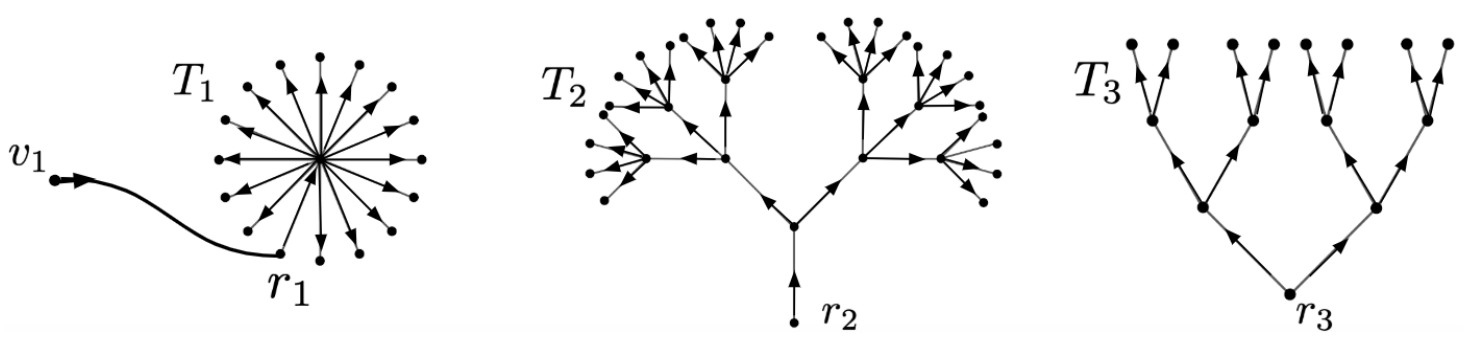

Figure 3.7: Subcase 1.
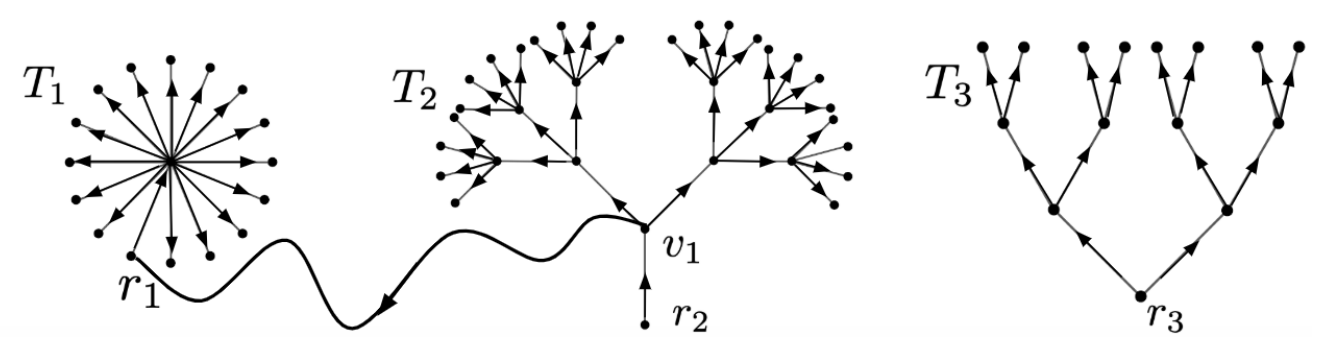

Figure 3.8: Subcase 2.

In this case, we have a contradiction on maximality since $F \cup\left(v_{1} \rightarrow r_{1}\right)$ is a forest and it has more edges than $F$ itself.※

Subcase 2: The vertex $v_{i} \in T_{i}$ for some $i \neq 1$. As shown in figure 3.8, for example.

Similarly, this is contradiction on maximality.

Subcase 3: The vertex $v_{i} \in T_{1}$. As shown in figure 4.1, for example.

This is a contradiction on cyclicity, as this would form a directed cycle. Thus, if $\Gamma$ is a directed acyclic graph, then $\operatorname{DF}(\Gamma)$ is shellable.

Q.E.D. 

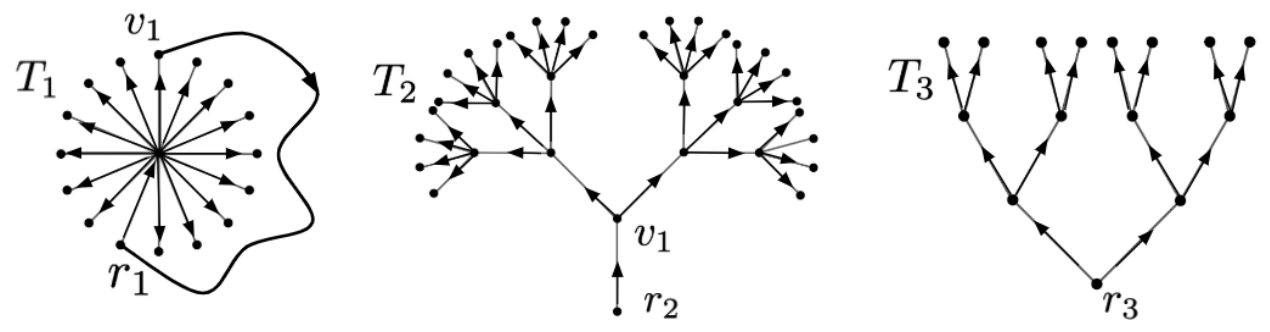

Figure 3.9: Subcase 3. 


\section{Chapter 4}

\section{THE DIRECTED FOREST COMPLEX OF A CAYLEY GRAPH}

\subsection{Cayley graphs}

Definition 4.1.1. Given a group $G$ and a generating set $X$. We define the Cayley graph $\Gamma=\Gamma(G, X)=(G, G \times X, i, t)$ where $i(g, x)=g$ and $t(g, x)=g x$.

Note that, using the notation of the previous chapter, we have $(g, x)=(g \rightarrow g x)$. The group $G$ acts on $\Gamma$ via left multiplication: $g^{\prime} \cdot g=g^{\prime} g$, and $g^{\prime} \cdot(g, x)=\left(g^{\prime} g, x\right)$. This action is "continuous" in the following sense: $i\left(g^{\prime} \cdot(g, x)\right)=g^{\prime} \cdot i(g, x)$ and $t\left(g^{\prime} \cdot(g, x)\right)=g^{\prime} \cdot t(g, x)$. It induces a continuous (or simplicial) action on the directed forest complex $\operatorname{DF}(\Gamma)$ and we will say more about this later. It is common to label the edge $(g, x)$ with just $x$, if it is clear where it starts.

Example 4.1.1. Let $G=D_{3}=\left\langle a, b \mid a^{2}, b^{2},(a b)^{3}\right\rangle$. Then the Cayley graph $\Gamma=$ $\Gamma\left(D_{3},\{a, b\}\right)$ can be see in figure 4.2

Example 4.1.2. Let $G=\mathbb{Z}_{3} \times \mathbb{Z}_{3}=\left\langle x, y \mid x^{3}, y^{3}, x y x^{-1} y^{-1}\right\rangle$. Then the Cayley graph $\Gamma=\Gamma\left(\mathbb{Z}_{3} \times \mathbb{Z}_{3},\{x, y\}\right)$ is seen in figure 4.3 .

Note that this graph is not planar but can be drawn on a torus.

Definition 4.1.2. A Hamiltonian cycle in a Cayley graph is a simple closed edge loop that goes through every vertex exactly once. So if $G=\left\{g_{1}, \ldots, g_{n}\right\}$ and $X$ is a 


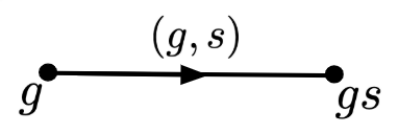

Figure 4.1: General edge in a Cayley graph

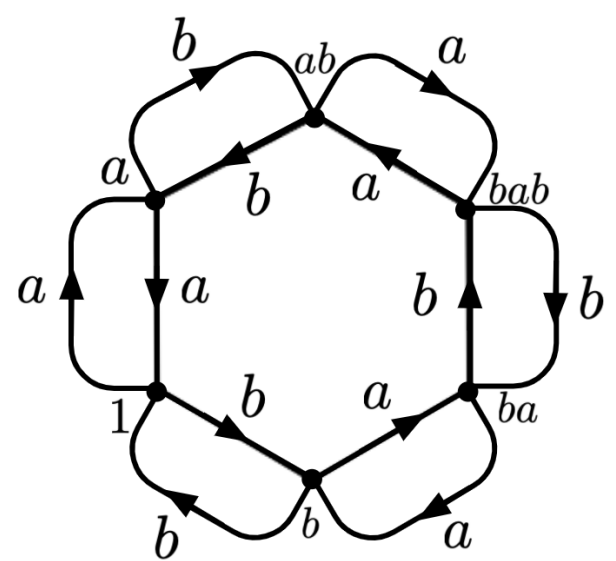

Figure 4.2: $\Gamma=\Gamma\left(D_{3},\{a, b\}\right)$

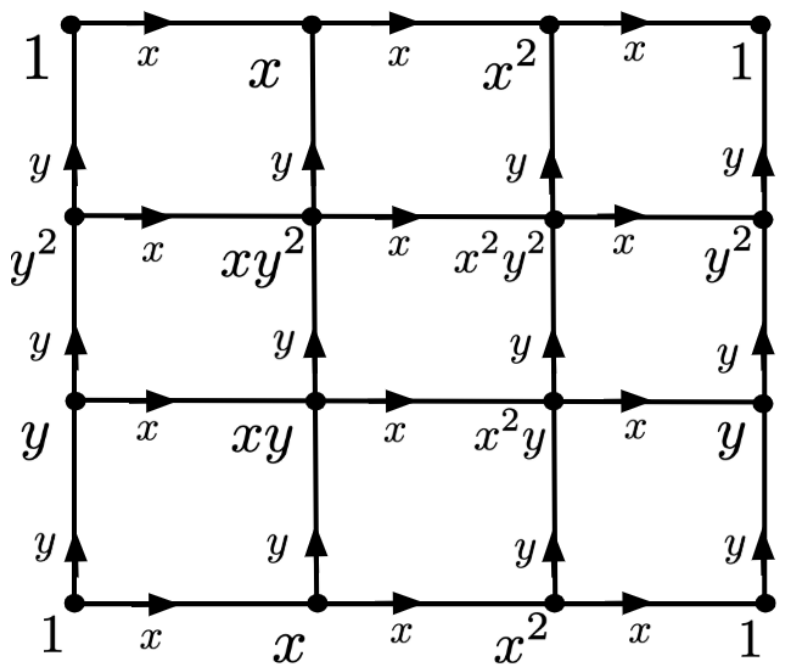

Figure 4.3: $\Gamma=\Gamma\left(\mathbb{Z}_{3} \times \mathbb{Z}_{3},\{x, y\}\right)$ 
generating set then a Hamiltonian cycle is of the form $\left(g_{1}, x_{1}\right)\left(g_{2}, x_{2}\right) \ldots\left(g_{n}, x_{n}\right)$, where $x_{i} \in X, g_{i} x_{i}=g_{i+1}$, and $g_{n} x_{n}=g_{1}$.

The following question is open:

Question. Do all Cayley graphs admit a Hamiltonian cycle? (Curran-Gallian [4])

The following however is known.

Theorem 4.1.1. (Pak-Radoicic [8]) For every finite group G there exists a generating set $X$ so that the associated Cayley graph does admit a Hamiltonian path.

What motivated some of this work is the fact that the homology of the directed forest complex can see Hamiltonian cycles.

Theorem 4.1.2. Every Hamiltonian cycle in a Cayley graph $\Gamma$ gives a nontrivial element in $H_{|G|-2}(\mathrm{DF}(\Gamma))$.

Proof. Suppose $|G|=n$ and $e_{1} \ldots e_{n}$ is a Hamiltonian cycle in $\Gamma$. Let $\sigma=\left\{e_{1}, \ldots, e_{n}\right\}$. Now $\sigma$ is not a simplex in $\operatorname{DF}(\Gamma)$, but its boundary $\partial \sigma$ is a subcomplex of $\operatorname{DF}(\Gamma)$. Note that $\sigma$ has dimension $|G|-1$, so $\partial \sigma$ has dimension $|G|-2$. Since $\partial \partial \sigma=0$, $\partial \sigma$ defines a non-trivial cycle in the top dimension, and hence a non-trivial homology element.

Q.E.D.

When we started work on this thesis, our hope was: Let $\Gamma$ be a Cayley graph. Then $\operatorname{DF}(\Gamma)$ is shellable and the dimension of the top dimensional homology equals the number of Hamiltonian cycles in $\Gamma$. In retrospect this seems naive, but sometimes it is true.

Example 4.1.3. Consider the Cayley graph of $\Gamma\left(\mathbb{Z}_{4}\right)$ in figure 4.4 . 


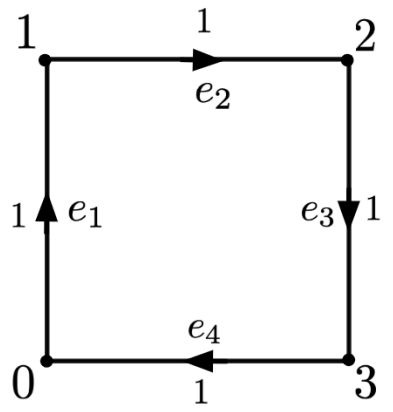

Figure 4.4: Cayley graph $\Gamma\left(\mathbb{Z}_{4}\right)$.

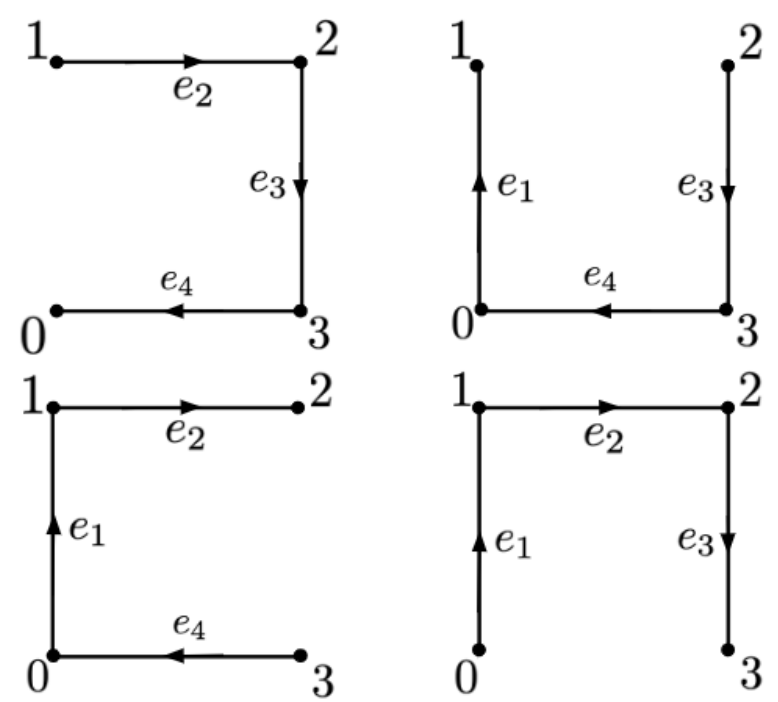

Figure 4.5: Top dimensional forests of $\mathrm{DF}\left(\mathbb{Z}_{4}\right)$. 


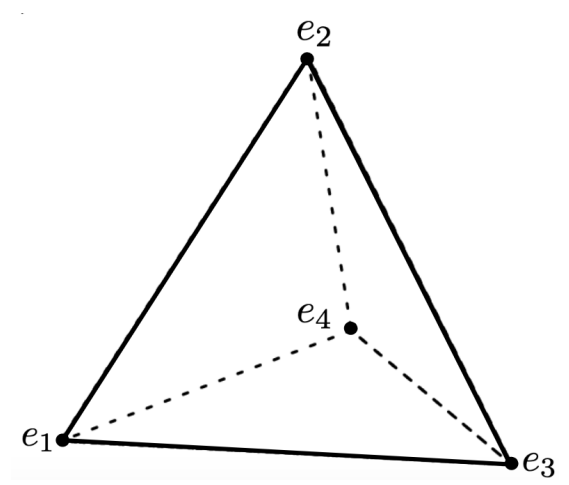

Figure 4.6: The geometric realization of $\mathrm{DF}\left(\mathbb{Z}_{4}\right)$.

Notice, this graph has a Hamiltonian cycle. We can draw the top dimensional forests which correspond to facets in the simplicial complex in figure 4.5.

Because each facet has three vertices, they are all realized geometrically by a (filled in) triangle. We can see the geometric realization of this complex as the boundary of a 3-simplex.

In this example, we see that $\operatorname{DF}(\Gamma)$ is shellable (we can choose any ordering of the facets in the boundary of a simplex to get a shelling order), and we see that the unique Hamilton cycle in the graph generates the unique homology element in $\operatorname{DF}(\Gamma)$.

More generally the dream theorem is true in the following setting.

Theorem 4.1.3. Let $G=\mathbb{Z}_{n}=\left\langle x \mid x^{n}=1\right\rangle$, and let $\Gamma$ be the associated Cayley complex. Then $\operatorname{DF}(\Gamma)$ is the boundary of an $(n-1)$-simplex, and thus is shellable. The dimension of $H_{n-2}(\mathrm{DF}(\Gamma))$ is one and $\Gamma$ contains exactly one Hamiltonian cycle.

In general the situation is more complicated. The next result is due to Koslov [7]. He studied (among other things) the homotopy type of the directed forest complex for double directed circles. 
Theorem 4.1.4. Let $G=D_{m}=\left\langle x, y \mid x^{2}=y^{2}=(x y)^{m}=1\right\rangle$ be the dihedral group and $\Gamma$ the associated Cayley graph. Then $\mathrm{DF}(\Gamma)$ is homotopic to:

$$
\begin{gathered}
S^{2 k-1} \vee S^{2 k-1} \vee S^{3 k-2} \vee S^{3 k-2} \text { if } 2 m=3 k, \\
S^{2 k} \vee S^{3 k-1} \vee S^{3 k-1} \text { if } 2 m=3 k+1, \\
S^{2 k} \vee S^{3 k} \vee S^{3 k} \text { if } 2 m=3 k+2 .
\end{gathered}
$$

Proof. Note that $\Gamma$ is a double directed cycle as considered in Koslov [7] and the result follows from his Proposition 5.2.

Q.E.D.

Note that in case of $D_{2}$, so $2 m=4=3 \cdot 1+1$, we get that $\operatorname{DF}(\Gamma)$ has the homotopy type of $S^{2} \vee S^{2} \vee S^{2}$. We will see this directly in the next chapter, where we aslo show that in that case $\operatorname{DF}(\Gamma)$ is shellable. Using a computer we computed the homology of $\mathrm{DF}(\Gamma)$ in the case of $D_{3}$ and found that the Betti vector for $H_{*}(\mathrm{DF}(\Gamma), \mathbb{R})$ is $\langle 1,0,0,2,2\rangle$. We did this before we knew Koslov's result that gives the homotopy type $S^{3} \vee S^{3} \vee S^{4} \vee S^{4}$, which matches with our computation. Note that this implies that $\mathrm{DF}(\Gamma)$ for $D_{3}$ is not shellable: because $\mathrm{DF}(\Gamma)$ is pure of dimension 4 (see next section) shellability would imply that there is no 3-dimensional homology.

\subsection{The question of purity}

Theorem 4.2.1. Let $\Gamma$ be the Cayley graph of a finite group. Then

1. A maximal directed forest in $\Gamma$ contains all the vertices of $\Gamma$;

2. Every directed tree $T$ is contained in a maximal directed tree that contains all the vertices of $\Gamma$. 
Proof. 1. Let $F$ be a maximal directed forest in a Cayley graph $\Gamma$. Suppose for the sake of contradiction that there exists a vertex $v \in \Gamma$ that is not contained in $F$. Let $e$ be an edge directed to $v$. We know $e$ exists because $\Gamma$ is a Cayley graph, and so at every vertex, there is at least one outgoing and one incoming edge. The edge $e$ is either disjoint from $F$ or connects to a component in $F$. In either case $e \cup F$ is a larger directed forest in $\Gamma$ that contains $F$, contradicting maximality.

2. Suppose that $T$ a directed tree in $\Gamma$ that is not contained in a bigger directed tree. We will show that $T$ contains all vertices of $\Gamma$. Let $r$ be the root of $T$. Suppose that $v$ is a vertex not contained in $T$. Note that there is a shortest directed path $\gamma$ in $\Gamma$ from $r$ to $v$. This is because $\Gamma$ is the Cayley graph of a finite group: if $x$ is a generator, then $x^{n}=1$ for some $n>1$ and so $x^{-1}=x^{n-1}$. So all elements $g \in G$ can be expressed as positive words in the generators. If $\gamma$ does not intersect $\Gamma$ except at $r$, then $T \cup \gamma$ is a bigger directed tree containing $T$. If $\gamma$ does intersect $T$, let $w$ be the last vertex on $\gamma \cap T$, and let $\gamma^{\prime}$ be the tail end of $\gamma$ that connects $w$ to $v$. Then $T \cup \gamma^{\prime}$ is a bigger directed tree.

Q.E.D.

Corollary 4.2.2. If $\Gamma$ is a Cayley graph then the dimension of $\operatorname{DF}(\Gamma)$ is $|G|-2$. Furthermore, $\mathrm{DF}(\Gamma)$ is pure if and only if all the facets come from maximal directed trees.

Proof. Let $\left\{e_{0}, \ldots, e_{n}\right\}$ be a facet of $\mathrm{DF}(\Gamma)$ and let $F=\bigcup_{i} e_{i}$ be the associated directed forest. By Theorem 4.2.1, $F$ contains all the vertices of $\Gamma$. Let $c$ be the number of components of $F$. We have

$$
c=\chi(F)=|G|-(n+1)
$$




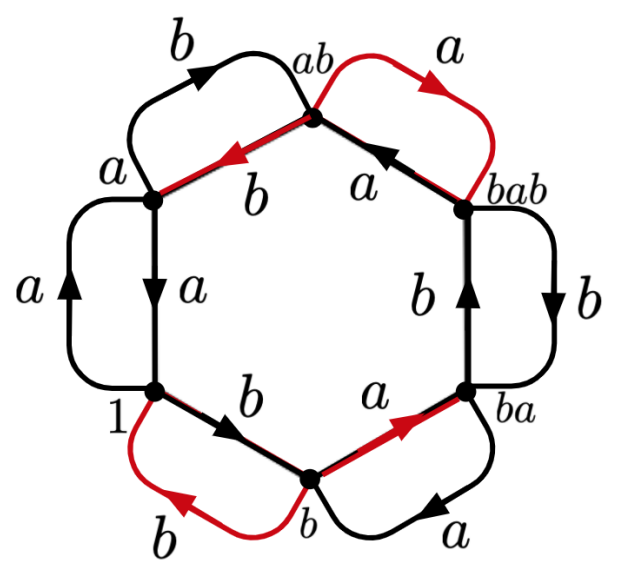

Figure 4.7: The Cayley graph of $D_{3}=\left\langle a, b \mid a^{2}, b^{2},(a b)^{3}\right\rangle$.

so $n=|G|-c-1$. Thus $n \leq|G|-2$. By Theorem 4.2.1 there exists a facet such that $F$ is a tree, in which case $c=1$ and $n=|G|-2$. This also shows that all facets have the same dimension $|G|-2$ if and only if all facets come from trees.

Q.E.D.

Not all $\operatorname{DF}(\Gamma)$ are pure. Consider figure 4.7 showing the Cayley graph of

$$
D_{3}=\left\langle a, b \mid a^{2}, b^{2},(a b)^{3}\right\rangle
$$

Note that there is a maximal directed tree containing 5 edges, so $\mathrm{DF}(\Gamma)$ contains a 4-dimensional facet. The red edges shown in the picture show a maximal directed forest (no edge can be added to make it a bigger directed forest) containing only 4 edges. So $\mathrm{DF}(\Gamma)$ contains a 3-dimensional facet.

\subsection{Actions and stabilizers}

The action of $G$ on a Cayley graph $\Gamma$ (by left multiplication) induces an action of $\operatorname{DF}(\Gamma)$. In this and the next sections we address the question of stabilizers and 
connectedness properties of $\mathrm{DF}(\Gamma)$.

We recall some relevant definitions. A action of a group on a set $X$ is a homomorphism $\phi: G \rightarrow \operatorname{Bij}(X)$, where $\operatorname{Bij}(X)$ is the group of bijections. We write $g x$ instead of $\phi(g)(x)$ in case the action is understood.

If $\Delta$ is a simplicial complex then a homeomorphism $f: \Delta \rightarrow \Delta$ is a bijection $f: V \rightarrow V$ on the set of vertices so that if $\left\{v_{0}, \ldots, v_{k}\right\}$ is a simplex then so is $\left\{f\left(v_{0}\right), \ldots, f\left(v_{k}\right)\right\}$. An action of $G$ on $\Delta$ is a group homomorphism $\phi: G \rightarrow H o m e o(\Delta)$, where $\operatorname{Homeo}(\Delta)$ is the group of homeomorphisms of $\Delta$. If $\sigma$ is a simplex we write $g \sigma$ instead of $\phi(g)(\sigma)$. The orbit of a simplex $\sigma$ is the set

$$
G \sigma=\{g \sigma \mid g \in G\}
$$

The stabilizer of a simplex $\sigma$ is the subgroup

$$
\operatorname{stab}(\sigma)=\{g \in G \mid g \sigma=\sigma\}
$$

We say a group acts freely on a set if all stabilizers stab(x) are trivial.

As always, let $G$ be a finite group, $\Gamma$ be a Cayley graph for $G$ associated with a generating set, and $\operatorname{DF}(\Gamma)$ be the directed forest complex.

Theorem 4.3.1. G acts freely on the vertices of $\mathrm{DF}(\Gamma)$.

Proof. The vertices of $\operatorname{DF}(\Gamma)$ are the edges in $\Gamma$. The group $G$ acts freely on $\Gamma$, in particular on its edge set: $g^{\prime}(g, x)=\left(g^{\prime} g, x\right)=(g, x)$ implies that $g^{\prime} g=g$, and it follows that $g^{\prime}=1$

Q.E.D. 
The next result is general and applies outside the strict setting of directed forest complexes. Let $G$ be a finite group and $\Delta$ be a simplicial complex on which $G$ acts.

Theorem 4.3.2. Let $k$ be the smallest number so that there exists a $k$-simplex, $\sigma$, so that the stabilizer of $\sigma$ is not trivial. Then $k+1$ divides the order of $G$.

Proof. Let $\sigma=\left\{v_{0}, \ldots, v_{k}\right\}$. Let $H$ be the stabilizer of $\sigma$ and let $h$ be a non-trivial element of order $m$ of $H$. Consider the orbit

$$
\langle h\rangle\left\{v_{0}\right\}=\left\{v_{0}, h v_{0}, h^{2} v_{0}, \ldots, h^{m-1} v_{0}\right\} \subseteq \sigma=\left\{v_{0}, \ldots v_{k}\right\}
$$

This orbit has to be equal to $\sigma$ by the assumption on $k$ : if the orbit is properly contained in $\sigma$ then $h$ is contained in the stabilizer of a proper face of $\sigma$, but we assumed the stabilizers of such are trivial. It follows that $m=k+1$, where $m$ is the order of $h$. So $k+1$ divides the order of $H$ and hence the order of $G$. Q.E.D. Example 4.3.1. Let $G=\mathbb{Z}_{p}$, where $p$ is a prime. Let $\Gamma$ be a Cayley graph of $G$ coming from generating set with a single element. Then $\Gamma$ is a single $p-c y c l e$. Then $\operatorname{DF}(\Gamma)$ is the boundary of a $p-1$ simplex (topologically a $p-2$-sphere), and $G$ acts freely on $\operatorname{DF}(\Gamma)$.

Example 4.3.2. In case $G=\mathbb{Z}_{n}$, where $n$ is not a prime we still have a Cayley graph that is a single $n$-cycle, and $\operatorname{DF}(\Gamma)$ is still the boundary of an $n-1$-simplex, but the stabilizers are not trivial anymore. For example, if $G=\mathbb{Z}_{4}=\langle x\rangle$, then the stabilizer of $\left\{(1, x),\left(x^{2}, x\right)\right\}$ contains $x^{2}$.

Example 4.3.3. Let $G=\mathbb{Z}_{5} \times \mathbb{Z}_{5}$, then $\operatorname{dim}(\operatorname{DF}(\Gamma))=23$. We know that $G$ acts freely on the 0 -skeleton of $\operatorname{DF}(\Gamma)$. Now $1+1=2,2+1=3,3+1=4$ do not divide the order of $G$ which is 25 . Thus $G$ acts freely on the 3 -skeleton of $\operatorname{DF}(\Gamma)$. Note that 
$4+1=5$ does divide the order of $G$, so $G$ might stabilize a 4-simplex (but we can not be sure without checking).

The following theorem is due to Serre.

Theorem 4.3.3. If a group $G$ acts orientation-preserving on a tree $T$, then it fixes a vertex.

Proof. We will sketch a proof in the case that $G$ and $T$ are finite. Let $L$ be a longest line in $T$, and let $v$ be its midpoint. Then $g v$ is the midpoint of the line $g L$. Choose a connecting line $L_{0}$ from $v$ to $g v$, then half of $L$ together with $L_{0}$ and half of $g L$ is a line that is longer than $L$ unless the length of $L_{0}$ is 0 . Thus, $v=g v$.

Q.E.D.

Theorem 4.3.4. Let $F=\left\{e_{0}, \ldots, e_{n}\right\}$ be a facet of $\mathrm{DF}(\Gamma)$ so that $T=\bigcup_{i} e_{i}$ is a tree. Then, $\operatorname{stab}(F)=\mathbb{1}$.

Proof. Let $g \in \operatorname{stab}(F)$, then $g F=F$. In particular, $\bigcup g e_{i}=\bigcup e_{i}$. Then, $g T=T$. By Serre's theorem, $g$ fixes a vertex of $T$. Hence, $g=1$, and therefore $\operatorname{stab}(F)=\mathbb{1}$.

Q.E.D.

We summarize the findings from this section. If $\Gamma$ is the Cayley graph of a finite group, then $G$ acts freely on the vertices and facets of $\mathrm{DF}(\Gamma)$ that come from directed trees. We also have the following divisibility condition: if $p$ is the smallest prime factor of $|G|$, then $G$ acts freely on simplices of dimension up to $p-2$. However, the action on larger simplices may fail to be free.

\subsection{Simple connectivity}

Let $G$ be a finite group and $\Gamma=\Gamma(G, X)$ a Cayley graph. Let $n=|X|$. 
Theorem 4.4.1. If for every four edges $e_{1}, e_{2}, e_{3}, e_{4} \in \Gamma$ there exists an edge e disjoint from the union of the $e_{i}$, then $\mathrm{DF}(\Gamma)$ is simply connected.

Proof. Suppose $\left\{e_{1}, e_{2}\right\},\left\{e_{2}, e_{3}\right\},\left\{e_{3}, e_{4}\right\}, \ldots,\left\{e_{m}, e_{1}\right\}$ is a simple edge loop in $\mathrm{DF}(\Gamma)$. Suppose that $m \geq 4$. Choose $e$ disjoint from the union $e_{1} \cup e_{2} \cup e_{3} \cup e_{4}$. Then we have 2-simplices $\left\{e_{i}, e_{i+1}, e\right\}, i=1,2,3$ in $\operatorname{DF}(\Gamma)$ and we can cone off the first 3 edges in the loop using the triangles. This gives a shorter loop $\left\{e_{1}, e\right\}\left\{e, e_{4}\right\}\left\{e_{4}, e_{5}\right\} \ldots\left\{e_{m}, e_{1}\right\}$. If $m=3$ (the smallest possible number) then we cone the entire loop off.

Q.E.D.

Corollary 4.4.2. If $|G|>16-\frac{4}{n}$, then $D F(\Gamma)$ is simply connected.

Proof. Consider four edges $e_{1}, \ldots, e_{4}$ in $\Gamma$. Note that there are $2 n-1$ edges distinct from $e_{1}$ at each vertex of $e_{1}$. Thus there are $2(2 n-1)+1$ edges connected to $e_{1}$ (including $\left.e_{1}\right)$. It follows that there can not be more than $4(4 n-1)=16 n-4$ edges

connected to the union $e_{1} \cup e_{2} \cup e_{3} \cup e_{1} \cup e_{4}$. Note that we have $n|G|$ edges in $\Gamma$. Thus if $n|G|>16 n-4$, then there is an edge $e$ in $\Gamma$ disjoint from the union.

Q.E.D.

Example 4.4.1. If $G=\mathbb{Z}_{5} \times \mathbb{Z}_{5}=\left\langle a, b \mid a^{5}=1, b^{5}=1, a b=b a\right\rangle$. Here $|G|=25>16$, so $\mathrm{DF}(\Gamma)$ is simply connected.

\subsection{A fundamental domain}

Suppose a group $G$ acts on a simplicial complex $\Delta$. A fundamental domain is a subcomplex $D$ which contains exactly one simplex from every orbit $G \sigma$, where $\sigma$ is a maximal simplex in $\Delta$.

Consider $\operatorname{DF}_{g}(\Gamma)$. Recall that this is the subcomplex of $\mathrm{DF}(\Gamma)$ whose facets come from trees in $\Gamma$ with root $g$, where $\Gamma$ is a Cayley graph. 
Theorem 4.5.1. $D F_{1}(\Gamma)$ is shellable and contractible.

Proof. Engström's Theorem 3.0.5 on rooted directed forest complexes gives shellability. We are left to show contractibility. Let $\left\{x_{1}, \ldots, x_{n}\right\}$ be the generating set used in building of $\Gamma$. We assume that all the $x_{i}$ are distinct. Let $e_{i}=\left(1, x_{i}\right)$. Note that $\left\{e_{1}, \ldots, e_{n}\right\}$ is a simplex in $\mathrm{DF}_{1}(\Gamma)$. Then every maximal tree in $\Gamma$ with root 1 contains at least one of the $e_{i}$. Let $K_{i}$ be the subcomplex of $\operatorname{DF}_{1}(\Gamma)$ where the maximal simplices contain $e_{i}$. Note that $K_{i}$ is the star of the vertex $e_{i}$ in $\operatorname{DF}_{1}(\Gamma)$ and so $K_{i}$ is contractible (see [6]). We have $\operatorname{DF}_{1}(\Gamma)=\bigcup K_{i}$. Note that $K_{i} \cap K_{j}$ is the subcomplex of $\mathrm{DF}_{1}(\Gamma)$ whose maximal simplices come from maximal trees that contain both $e_{i}$ and $e_{j}$. The set $\left\{e_{i}, e_{j}\right\}$ is an edge in $\mathrm{DF}_{1}(\Gamma)$, so the intersection $K_{i} \cap K_{j}$ is the star of that edge. Hence the intersection is contractible. Similarly, the intersections $K_{i} \cap K_{j} \cap K_{k}$ is the star of the simplex $\left\{e_{i}, e_{j}, e_{k}\right\}$, and so is contractible. This argument extends to all intersections, so all intersections are non-empty and contractible. Contractibility of $\mathrm{DF}_{1}(\Gamma)$ now follows from the nerve theorem (see Brown [?], Chapter VII, Theorem 4.4).

Q.E.D.

Lemma 4.5.2. If $\mathrm{DF}(\Gamma)$ is pure we have $\mathrm{DF}(\Gamma)=\bigcup_{g \in G} \operatorname{DF}_{g}(\Gamma)$.

Proof. We recall Corolary 4.2.2: $\mathrm{DF}(\Gamma)$ is pure if and only if all facets come from maximal directed trees in $\Gamma$. We can see that $\operatorname{DF}(\Gamma) \supseteq \bigcup_{g \in G} \operatorname{DF}_{g}(\Gamma)$ because each $\operatorname{DF}_{g}(\Gamma)$ is a subcomplex of $\operatorname{DF}(\Gamma)$. Now we will show that $\operatorname{DF}(\Gamma) \subseteq \bigcup_{g \in G} \operatorname{DF}_{g}(\Gamma)$. Suppose that $\sigma=\left\{e_{0}, \ldots e_{k}\right\}$ is a facet in $\operatorname{DF}(\Gamma)$. Then $T=\bigcup_{j=0}^{k} e_{j}$ is a maximal directed tree in $\Gamma$. Hence it has a root, say $g$. It follows that $\sigma \in \operatorname{DF}_{g}(\Gamma)$.

Q.E.D.

Lemma 4.5.3. $h \cdot \mathrm{DF}_{g}(\Gamma)=\mathrm{DF}_{h g}(\Gamma)$ 
Proof. Let $\sigma=\left\{e_{0}, \ldots, e_{k}\right\}$ be a facet in $\operatorname{DF}_{g}(\Gamma)$. Then $T=\bigcup e_{j}$ is a maximal directed tree with root $g$. Then $h T=\bigcup h e_{j}$ is a maximal directed tree with root $h g$. So, $h \sigma=\left\{h e_{0}, \ldots, h e_{k}\right\} \in \mathrm{DF}_{h g}(\Gamma)$.

Q.E.D.

The last two results give the following:

Corollary 4.5.4. $\mathrm{DF}_{1}(\Gamma)$ is a fundamental domain for the action of $G$ on $\operatorname{DF}(\Gamma)$ in case $\mathrm{DF}(\Gamma)$ is pure.

Question. If $\mathrm{DF}(\Gamma)$ is $n$ dimensional, then is it true that $\operatorname{DF}_{g}(\Gamma)$ is $n$ dimensional, $\mathrm{DF}_{g}(\Gamma) \cap \mathrm{DF}_{h}(\Gamma)$ is $n-1$ dimensional, $\mathrm{DF}_{g}(\Gamma) \cap \mathrm{DF}_{h}(\Gamma) \cap \mathrm{DF}_{f}(\Gamma)$ is $n-2$ dimensional, and so on...

Example 4.5.1. Let $G=D_{3}=\left\langle x, y \mid x^{2}, y^{2},(x y)^{3}\right\rangle$ and let $\Gamma$ be the associated Cayley graph. Using a computer we found that $H_{*}(\operatorname{DF}(\Gamma), \mathbb{R})=\langle 1,0,0,2,2\rangle$. The fact that the 3rd homology is not trivial might be a first hint that $\mathrm{DF}(\Gamma)$ is not pure. It might be non-pure shellable as Koslov's Theorem 4.1.4 seems to suggest.

One can attempt to obtain all homotopical information about $\mathrm{DF}(\Gamma)$ by trying to find a shelling order. That approach might be too optimistic, even if we asume $\operatorname{DF}(\Gamma)$ is pure. But in that case we can work with the cover $\operatorname{DF}(\Gamma)=\bigcup \operatorname{DF}_{g}(\Gamma)$. If intersections of the pieces $\mathrm{DF}_{g}(\Gamma)$ are shellable, then we know the homotopy type of the pieces and their intersections and thus can compute the homology of $\operatorname{DF}(\Gamma)$ using the Mayer-Vietoris spectral sequence.

Example 4.5.2. Let $\Gamma$ be the Cayley graph for $G=\mathbb{Z}_{2} \times Z_{2}=\left\langle x, y \mid x^{2}, y^{2},(x y)^{2}\right\rangle$, seen in figure 4.8 . 


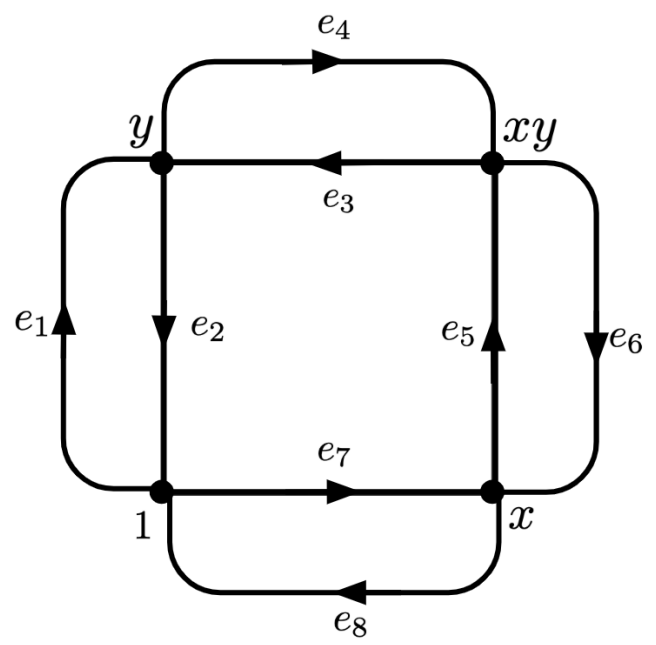

Figure 4.8: $\Gamma$, the Cayley graph for $G=\mathbb{Z}_{2} \times Z_{2}=\left\langle x, y \mid x^{2}, y^{2},(x y)^{2}\right\rangle$
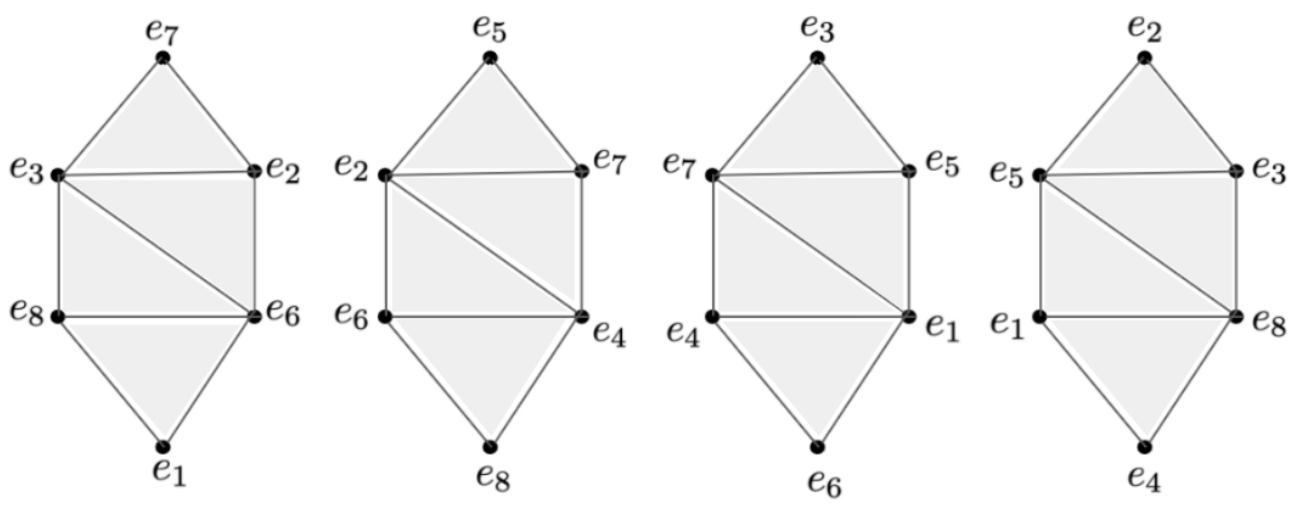

$\mathrm{DF}_{x y}(\Gamma)$

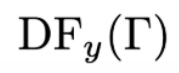

$\mathrm{DF}_{1}(\Gamma)$

$\mathrm{DF}_{x}(\Gamma)$

Figure 4.9: $\mathrm{DF}_{x y}(\Gamma) \mathrm{DF}_{y}(\Gamma) \mathrm{DF}_{1}(\Gamma), \mathrm{DF}_{x}(\Gamma)$ 


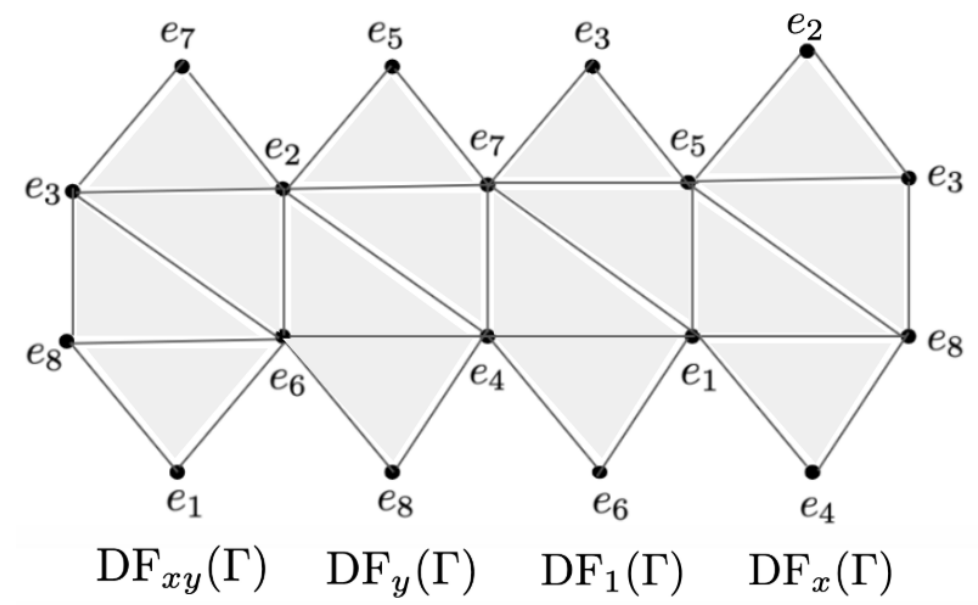

Figure 4.10: Some identifications made on the $\operatorname{setDF}_{x y}(\Gamma) \operatorname{DF}_{y}(\Gamma) \operatorname{DF}_{1}(\Gamma)$, $\mathrm{DF}_{x}(\Gamma)$.

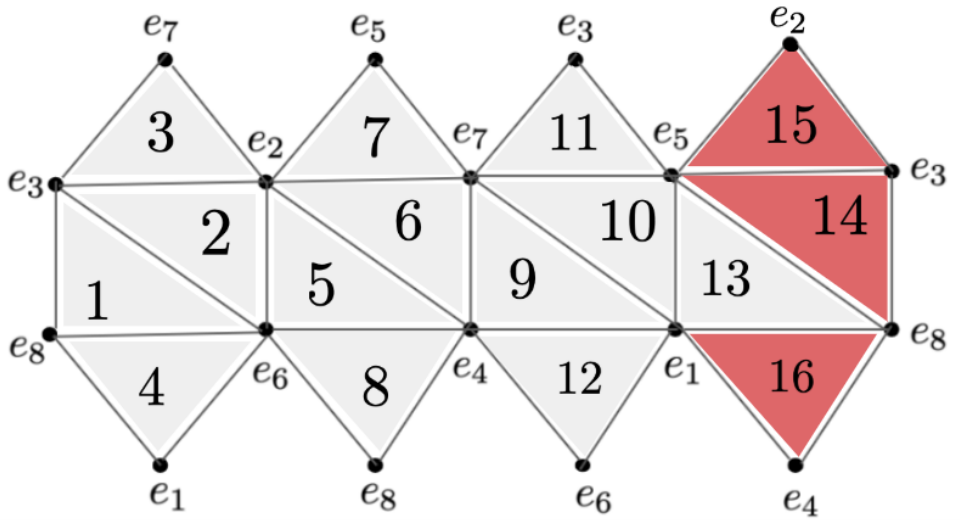

Figure 4.11: The capping forests. 
From this, we can draw $\mathrm{DF}_{x y}(\Gamma) \mathrm{DF}_{y}(\Gamma) \mathrm{DF}_{1}(\Gamma), \mathrm{DF}_{x}(\Gamma)$ respectively as seen in figure 4.9 .

Consider the following intersections,

$$
\begin{aligned}
\operatorname{DF}_{1}(\Gamma) \cap \mathrm{DF}_{x}(\Gamma) & =\left\{\left\{e_{3}, e_{5}\right\},\left\{e_{1}, e_{5}\right\},\left\{e_{1}, e_{4}\right\}\right\} \\
\operatorname{DF}_{1}(\Gamma) \cap \operatorname{DF}_{y}(\Gamma) & =\left\{\left\{e_{5}, e_{7}\right\},\left\{e_{4}, e_{6}\right\},\left\{e_{4}, e_{7}\right\}\right\} \\
\operatorname{DF}_{1}(\Gamma) \cap \mathrm{DF}_{x y}(\Gamma) & =\left\{\left\{e_{1}, e_{6}\right\},\left\{e_{3}, e_{7}\right\}\right\} \\
\mathrm{DF}_{1}(\Gamma) \cap \operatorname{DF}_{x}(\Gamma) \cap \mathrm{DF}_{y}(\Gamma) & =\left\{\left\{e_{4}\right\},\left\{e_{5}\right\}\right\}
\end{aligned}
$$

Knowing the intersections (all are indeed shellable) we could compute the homology using Mayer-Vietoris. But note that the complex $\operatorname{DF}(\Gamma)$ is shellable, and hence homotopically a wedge of 2 -spheres. A shelling order is indicated in the picture. The 3 red 2-simplices shown are the "capping simplices", the ones that produce the 2-spheres in the wedge. Note furthermore that $\Gamma$, being a double directed cycle, contains exactly two Hamiltonian cycles, but the dimension of $H_{2}(\mathrm{DF}(\Gamma), \mathbb{R})$ is 3 .

Intersections of the various $\mathrm{DF}_{g}(\Gamma)$ are difficult to understand. The above example also shows that in general $\mathrm{DF}_{1}(\Gamma) \cap \mathrm{DF}_{g}(\Gamma) \neq \mathrm{DF}_{\{1, g\}}(\Gamma)$.

Computation 1. The Betti vector of the directed forest complex of the Cayley graph $\mathbb{Z}_{2} \times \mathbb{Z}_{2}=D_{2}=\left\langle x, y \mid x^{2}, y^{2},(x y)^{2}\right\rangle$ is $\langle 1,0,3\rangle$. In fact, we know that this is complex is shellable.

Computation 2. The Betti vector of the directed forest complex of the Cayley graph 
$\mathbb{Z}_{3} \times \mathbb{Z}_{3}=\left\langle x, y \mid x^{3}, y^{3},(x y)^{3}\right\rangle$ is $\langle 1,0,0,0,0,0,0,28\rangle$. We believe $\mathrm{DF}(\Gamma)$ is pure in this case. We do not know if this complex is shellable.

Computation 3. The Betti vector of the directed forest complex of the Cayley graph $D_{3}=\left\langle x, y \mid x^{2}, y^{2},(x y)^{3}\right\rangle$ is $\langle 1,0,0,2,2\rangle$. We know that $\operatorname{DF}(\Gamma)$ is not pure. Is this detected by the homology? Is $\mathrm{DF}(\Gamma)$ non-pure shellable?

Theorem 4.5.5. If $\Gamma$ is the Cayley graph of a group $G$ such that $G \neq \mathbb{Z}_{2}$, then $\mathrm{DF}_{g_{1}}(\Gamma) \cap \mathrm{DF}_{g_{2}}(\Gamma) \neq \emptyset$ for every $g_{1}, g_{2} \in G$.

Proof. It suffices to show that $\mathrm{DF}_{1}(\Gamma) \cap \mathrm{DF}_{g}(\Gamma) \neq \emptyset$ for every $g \in G$. If $G$ is cyclic with minimal generating set then the statement is true as $\Gamma$ is a cycle of length 3 or greater. Now suppose $\Gamma$ arises from a generating set containing at least two generators. There exists an edge $e_{*}$ for which the the identity is the initial vertex and the element $g$ is not the terminal vertex. By definition, $e_{*} \in D F_{1}(\Gamma)$. We can connect the vertex $g$ to the vertex $e_{*}$ by a directed edge path $e_{1}, \ldots, e_{k}$ that starts at $g$ and ends at the identity element. Then $\left\{e_{1}, \ldots, e_{k}, e_{*}\right\} \in \mathrm{DF}_{g}(\Gamma)$. Thus, $e_{*} \in \mathrm{DF}_{1}(\Gamma) \cap \mathrm{DF}_{g}(\Gamma)$.

Q.E.D.

For the definition of Hamiltonian cycle see Definition 4.1.2. Here are some examples of Cayley graphs with directed Hamiltonian cycle:

$\Gamma$ for $\mathrm{D}_{n}=\left\langle x, y \mid x^{2}=1, y^{2}=1,(x y)^{n}=1\right\rangle$.

$\Gamma$ for

$$
\mathbb{Z}_{n} \times \mathbb{Z}_{m}=\left\langle x, y \mid x^{n}=1, y^{m}=1, x y=y x\right\rangle
$$

Lemma 4.5.6. Suppose $\Gamma$ is a Cayley graph that contains a directed Hamiltonian cycle. Then 
1. for every $x \in G, \bigcap_{g \in G, g \neq x} \mathrm{DF}_{g}(\Gamma) \neq \emptyset$, and

2. $\bigcap_{g \in G} \mathrm{DF}_{g}(\Gamma)=\emptyset$.

Proof. (1) Set $Z$ be a directed Hamiltonian cycle. Let $e$ be the edge in $Z$ that terminates at the vertex $x$, then $e \in \bigcap \operatorname{DF}_{g}(\Gamma)$. (2) Note that if $e$ is an edge in $\Gamma$ that terminates in $g$, then $\{e\} \notin \operatorname{DF}_{g}(\Gamma)$.

Q.E.D.

Definition 4.5.1. Suppose $X$ is a simplicial complex and $X=\bigcup_{j \in J} X_{j}$ is a cover of $X$ by subcomplexes. The nerve, $\mathrm{N}$, is a simplicial complex is defined as follows,

1. The set of vertices is $J$, and

2. The set $j_{0}, \ldots, j_{k}$ is a simplex if $X_{j_{0}} \cap \cdots \cap X_{j_{k}} \neq \emptyset$.

The nerve comes with a coefficient system. If $\sigma=\left\{j_{0}, \ldots, j_{k}\right\}$ is a simplex of $N$, we denote by $X_{\sigma}=X_{j_{0}} \cap \ldots \cap X_{j_{k}}$. Note that if $\tau \subseteq \sigma$ then we have a map $H_{q}\left(X_{\sigma}\right) \rightarrow H_{q}\left(X_{\tau}\right)$ induced by inclusion. We can define a chain complex

$$
\mathcal{H}_{q}: \ldots \rightarrow \bigoplus_{\sigma \in X} H_{q}\left(X_{\sigma}\right) \rightarrow \bigoplus_{\tau \in X} H_{q}\left(X_{\tau}\right) \rightarrow \ldots
$$

whose $p$ th homology we denote by $H_{p}\left(N, \mathcal{H}_{q}\right)$. The spectral sequence

$$
E_{p q}^{2}=H_{p}\left(N, \mathcal{H}_{q}\right) \Rightarrow H_{p+q}(X)
$$

is called the Mayer-Vietoris spectral sequence. Details on this can be found in Brown [3], Chapter VII. Thus, in order to compute $H_{n}(X)$ one needs to get hold of the nerve and understand the intersections of the various $X_{j}$. 
We complete this thesis by computing the nerve of the cover $\mathrm{DF}(\Gamma)=\bigcup_{g \in G} \mathrm{DF}_{g}(\Gamma)$ in case $\operatorname{DF}(\Gamma)$ is pure.

Theorem 4.5.7. Let $\Gamma$ be a Cayley graph with a directed Hamiltonian cycle. Assume that $\operatorname{DF}(\Gamma)$ is pure. The nerve $N$ of this covering, $\operatorname{DF}(\Gamma)=\bigcup \operatorname{DF}_{g}(\Gamma)$, is $\partial \Delta(G)$, where $\Delta(G)$ is the simplex with vertex set $G$. Thus $N$ is topologically a $(|G|-2)$ sphere.

Proof. Note that the nerve $N$ can be described as follows, vertices are elements of $G$ and the simplicies $\left\{g_{0}, \ldots, g_{k}\right\} \in N$ if $\mathrm{DF}_{g_{0}}(\Gamma) \cap \cdots \cap \mathrm{DF}_{g_{k}}(\Gamma) \neq \emptyset$. Then by Lemma 4.5.6, $N=\partial \Delta(G)$, where $\Delta(G)$ is the full simplex on vertex set $G$.

Q.E.D. 


\section{Bibliography}

[1] Anders Björner and Michelle Wachs. Shellable nonpure complexes and posets. i. Transactions of the American mathematical society, 348(4):1299-1327, 1996.

[2] Béla Bollobás. Modern graph theory, volume 184. Springer Science \& Business Media, 2013.

[3] Kenneth S Brown. Cohomology of groups, volume 87. Springer Science \& Business Media, 2012.

[4] Stephen J Curran and Joseph A Gallian. Hamiltonian cycles and paths in cayley graphs and digraphs - a survey. Discrete Mathematics, 156(1-3):1-18, 1996.

[5] Alexander Engström. Complexes of directed trees and independence complexes. Discrete Mathematics, 309(10):3299-3309, 2009.

[6] Dimitry Kozlov. Combinatorial algebraic topology, volume 21. Springer Science \& Business Media, 2007.

[7] Dmitry N Kozlov. Complexes of directed trees. Journal of Combinatorial Theory, Series A, 88(1):112-122, 1999.

[8] Igor Pak and Radoš Radoičić. Hamiltonian paths in cayley graphs. Discrete Mathematics, 309(17):5501-5508, 2009. 